The Astrophysical Journal, 213:737-755, 1977 May 1

(C) 1977. The American Astronomical Society. All rights reserved. Printed in U.S.A.

\title{
INFRARED PHOTOMETRY AND MASS LOSS RATES FOR OBA SUPERGIANTS AND Of STARS
}

Michael J. BARLOW

Joint Institute for Laboratory Astrophysics, University of Colorado and National Bureau of Standards

AND

MARTIN COHEN

Astronomy Department, University of California, Berkeley

Received 1976 August 2

\begin{abstract}
We present infrared observations of 34 OBA supergiants and 10 Of and Oe stars. Excess freefree emission is detectable at $10 \mu \mathrm{m}$ but not at shorter wavelengths for the supergiants. From our 2.2 and $3.6 \mu \mathrm{m}$ photometry of the supergiants, in conjunction with existing $U B V$ data, we derive an invariant ratio of total to selective extinction, $R=3.10 \pm 0.03$. Using published radio and infrared data, we derive the velocity law for the mass loss outflow from P Cygni, and we apply this velocity law to the measured $10 \mu \mathrm{m}$ excess fluxes from our sample of supergiants in order to derive mass loss rates. Our preliminary survey of Of and Oe stars shows excess free-free emission to be common at $3.6 \mu \mathrm{m}$ and sometimes detectable at $2.2 \mu \mathrm{m}$. Using our 2.2-10 $\mu \mathrm{m}$ data, we derive mass loss rates for several $\mathrm{O}$ stars. Our results are consistent with radiation pressure being the driving mechanism for mass loss from OBA supergiants and Of stars.
\end{abstract}

Subject headings: interstellar: matter - stars: circumstellar shells - stars: early-type stars: mass loss - stars: Of-type — stars: supergiants

\section{INTRODUCTION}

As part of an infrared investigation of early-type emission-line stars, we have observed 34 supergiants having spectral types in the range 09.5 to $\mathrm{A} 5$, using broad-band systems principally covering the wavelength range 2.2-10 $\mu \mathrm{m}$, with occasional observations at longer wavelengths $(\leq 18 \mu \mathrm{m})$ where possible. In addition, we have made a preliminary study of some bright Oe and Of stars, and we report observations of 10 such stars.

In $\S$ II, we present the observations of the supergiants. In $\S$ III, using the observations at 2.2 and $3.6 \mu \mathrm{m}$, we derive the value of $R\left[=A_{V} / E(B-V)\right]$ for the general interstellar medium. It is found that excess $10 \mu \mathrm{m}$ flux occurs for supergiants with requisite spectral type and luminosity. These results are discussed in $\S$ IV, and the excess flux is interpreted as arising from circumstellar free-free emission. In $\S \mathrm{V}$, we derive the velocity law for the mass loss outflow from P Cyg and apply this velocity law to the other supergiants in our sample in order to derive mass loss rates. In $\S$ VI, the observations and results are described for the Of and Oe stars. Finally, in $\S$ VII, we discuss the nature of the mechanism driving the observed mass loss flows.

\section{THE OBSERVATIONS}

All our infrared photometry, with the exceptions of that for the stars $\kappa$ Cas and HD 108, was obtained using a liquid-helium-cooled bolometer and a multifilter system on the UM/UCSD $1.5 \mathrm{~m}$ telescope on Mount Lemmon, Arizona. For these observations, 11" beams with 15" separation were used. Details of the multifilter system are given by Cohen (1973). The data for $\kappa$ Cas and HD 108 were acquired similarly, using $4 "$ beams separated by $8^{\prime \prime}$, at the Cassegrain focus of the Lick Observatory $3.05 \mathrm{~m}$ reflector. The observations spanned the period 1972 December-1974 April.

Table 1 presents our photometry for the supergiants as follows: column (1) gives the HD number and column (2) the name of the star; column (3) gives the MK spectral type; columns (4) and (5) give $V$ and $(B-V)$; and columns (6)-(12) present the infrared observations at the designated wavelengths in the form of magnitudes with parenthesized errors corresponding to $1 \sigma$ of the mean (in hundredths of a magnitude), or $3 \sigma$ upper limits in the case of no detection. Column (13) gives the number, $N$, of occasions on which each star was observed. Care was taken to ensure good coverage of each spectral type in the range 09.5-A2. However, no B4 supergiants were accessible from the northern hemisphere. The source of the $B$ and $V$ data was the catalog of Blanco et al. (1968). It was found that the entries for each supergiant typically had a range of $\sim 0.06$ mag for $V$ and about half this for the $(B-V)$ color. This range appears to be due to the small-amplitude photometric variability discovered by Maeder and Rufener (1972) for luminous early-type supergiants. We selected from the catalog the data set closest to the median value for each star. 
Table 1

\begin{tabular}{|c|c|c|c|c|c|c|c|c|c|c|c|c|}
\hline HD & Name & Sp. Type & $\mathrm{v}$ & $(B-V)$ & {$[2.2]$} & {$[3.6]$} & {$[4.8]$} & {$[8.6]$} & [10] & [11.3] & {$[18]$} & $\mathrm{N}$ \\
\hline 36486 & $\delta$ ori & $09.5 \mathrm{I}$ & 2.24 & -0.21 & $2.87 *$ & $2.87(01)$ & $2.95(08)$ & $2.96(18)$ & & $2.73(20)$ & $>0.22$ & 1 \\
\hline 37742 & $\zeta$ Ori & $09.5 \mathrm{I}$ & 1.74 & -0.21 & $2.32(06)$ & $2.28(01)$ & $2.32(06)$ & $2.25(08)$ & $2.30(05)$ & $2.42(08)$ & & 2 \\
\hline 37128 & $\varepsilon$ ori & BOIa & 1.70 & -0.19 & $2.22(05)$ & $2.23(01)$ & $2.22(05)$ & $2.21(08)$ & & $2.02(08)$ & $0.80(24)$ & 3 \\
\hline 38771 & к Ori & $\mathrm{B} 0.5 \mathrm{Ia}$ & 2.06 & -0.18 & $2.64(01)$ & $2.67(01)$ & $2.57(07)$ & $2.47(16)$ & & $2.45(16)$ & $>0.00$ & 2 \\
\hline 169454 & & $\mathrm{BlIa}^{+}$ & 6.61 & 0.94 & $4.03(02)$ & $3.81(02)$ & & & $3.46(18)$ & & & 1 \\
\hline 2905 & k Cas & $\mathrm{B} 1 \mathrm{Ia}$ & 4.16 & 0.13 & $3.93(01)$ & $3.79(01)$ & & & $3.55(06)$ & & & 1 \\
\hline 24398 & $\zeta$ Per & B1Ib & 2.85 & 0.12 & $2.57(01)$ & $2.54(01)$ & & & $2.54(03)$ & & & 4 \\
\hline 40111 & $139 \mathrm{Tau}$ & $\mathrm{B} 1 \mathrm{Ib}$ & 4.82 & -0.07 & $5.10(03)$ & $5.02(04)$ & & & & & & 1 \\
\hline 91316 & $\rho$ Leo & B1Iab & 3.85 & -0.13 & $4.23(01)$ & $4.23(01)$ & $4.23(32)$ & & $4.05(1.3)$ & & & 4 \\
\hline 190603 & & $\mathrm{~B} 1.5 \mathrm{Ia}^{+}$ & 5.65 & 0.55 & $4.03(01)$ & $3.95(02)$ & & & $3.55(12)$ & & & 2 \\
\hline 194279 & & $\mathrm{~B} 1.5 \mathrm{Ia}$ & 7.03 & 1.02 & $4.21(01)$ & $3.96(02)$ & & & $3.59(20)$ & & & 1 \\
\hline 14143 & & B2Ia & 6.66 & 0.50 & $5.28(04)$ & $5.13(04)$ & & & $4.90(30)$ & & & 2 \\
\hline 41117 & $x^{2}$ ori & B2Ia & 4.63 & 0.27 & $3.89(01)$ & $3.69(01)$ & $3.75(18)$ & & $3.45(10)$ & & & 2 \\
\hline 14134 & & B3Ia & 6.55 & 0.47 & $5.32(01)$ & $5.13(01)$ & & & $4.88(23)$ & & & 3 \\
\hline 53138 & $\circ^{2} \mathrm{CMa}$ & B3Ia & 3.04 & -0.08 & $3.33(01)$ & $3.19(02)$ & & & $2.76(10)$ & & & 1 \\
\hline 198478 & 55 Cyg & B3Ia & 4.83 & 0.40 & $3.84(01)$ & $3.78(01)$ & $3.71(14)$ & & $3.40(10)$ & & & 2 \\
\hline 58350 & $\mathrm{n} \mathrm{CMa}$ & B5Ia & 2.44 & -0.09 & $2.79(01)$ & $2.69(02)$ & & & $2.57(08)$ & & & 2 \\
\hline 36371 & $x$ Aur & B5Iab & 4.77 & 0.35 & $3.75(01)$ & $3.72(01)$ & & & $3.26(10)$ & & & 2 \\
\hline 164353 & 67 oph & B5Ib & 3.96 & 0.02 & $3.96(01)$ & $3.87(02)$ & & & $3.82(25)$ & & & 1 \\
\hline 15497 & & B6Ia & 7.03 & 0.78 & $4.95(03)$ & $4.77(02)$ & & & $4.46(17)$ & & & 2 \\
\hline 183143 & & B7 Ia & 6.86 & 1.22 & $3.46(01)$ & $3.20(01)$ & & & $3.14(10)$ & & & 2 \\
\hline 34085 & B Ori & B8Ia & 0.15 & -0.03 & $0.09(01)$ & $0.10(01)$ & $0.05(01)$ & $0.00(04)$ & $0.06(02)$ & $0.04(03)$ & $-0.31(17)$ & 2 \\
\hline 166937 & $\mu \mathrm{Sgr}$ & B8Ia & 3.86 & 0.24 & $3.19(01)$ & $3.08(01)$ & $3.19(17)$ & & $2.98(10)$ & & & 3 \\
\hline 208501 & $13 \mathrm{Cep}$ & B8Ib & 5.81 & 0.71 & $3.91(01)$ & $3.70(01)$ & & & $3.65(16)$ & & & 2 \\
\hline 21291 & & B9Ia & 4.21 & 0.41 & $3.01(01)$ & $2.92(01)$ & & & $2.84(08)$ & & & 1 \\
\hline 202850 & $\sigma$ Cyg & B9Iab & 4.22 & 0.11 & $3.82(01)$ & $3.90(02)$ & & & $3.94(18)$ & & & 2 \\
\hline 212953 & 4 Lac & B9Iab & 4.57 & 0.08 & $4.39(01)$ & $4.30(01)$ & & & $4.37(19)$ & & & 3 \\
\hline 21389 & & $\mathrm{AOIa}$ & 4.55 & 0.56 & $2.85(01)$ & $2.77(01)$ & & & $2.57(06)$ & & & 2 \\
\hline 87737 & $n$ Leo & $\mathrm{AOIb}$ & 3.53 & -0.04 & $3.39(01)$ & $3.38(01)$ & & & $3.34(08)$ & & & 3 \\
\hline 12953 & & AlIa & 5.68 & 0.62 & $3.92(01)$ & $3.69(01)$ & & & $3.56(08)$ & & & 3 \\
\hline 14489 & 9 Per & $\mathrm{A} 2 \mathrm{Ia}$ & 5.17 & 0.37 & $4.05(01)$ & $3.96(01)$ & & & $3.83(12)$ & & & 2 \\
\hline 197345 & $\alpha$ Cyg & $\mathrm{A} 2 \mathrm{Ia}$ & 1.21 & 0.11 & $0.93(01)$ & $0.83(01)$ & $0.84(01)$ & $0.70(01)$ & $0.69(01)$ & $0.67(01)$ & $0.09(25)$ & 3 \\
\hline 207260 & $v$ Cep & A2Ia & 4.29 & 0.51 & $2.86(01)$ & $2.75(02)$ & & & $2.91(09)$ & & & 1 \\
\hline 17378 & & A5 Ia & 6.26 & 0.89 & $3.79(01)$ & $3.58(03)$ & & & $3.47(13)$ & & & 1 \\
\hline
\end{tabular}

*From Lee $(1968)$.

\section{INFRARED COLORS AND THE INTERSTELLAR EXTINCTION LAW}

Plots of the observed $(B-V)$ color indices versus the observed $(V-K)$ and $(V-L)$ indices (where $K$ denotes [2.2], the $2.2 \mu \mathrm{m} \mathrm{mag}$, and $L$ denotes the $3.6 \mu \mathrm{m} \mathrm{mag}$ ) are shown in Figures 1 and 2 for 33 supergiants. We have omitted $\beta$ Ori because of the unusually discrepant $U B V$ data (a range of $0.22 \mathrm{mag}$ at $V$ ). Both Figures 1 and 2 show striking correlations. Least-squares solutions with equal weight taking $(B-V)$ as the independent variable yield the following relationships for the observed colors:

$$
\begin{aligned}
(V-K) & =(2.83 \pm 0.04)(B-V)-(0.04 \pm 0.02), \\
(V-L) & =(2.96 \pm 0.03)(B-V)+(0.04 \pm 0.02) .
\end{aligned}
$$

These relations may be used to predict $K$ and $L$ magnitudes, independent of any knowledge of intrinsic colors or the interstellar reddening law.

Using our infrared data, we may now attempt to derive the ratio of total to selective extinction, $R$. The $10 \mu \mathrm{m}$ observations will not be used to this end because, as discussed in detail later in this section and in $\S$ IV, many supergiants show $10 \mu \mathrm{m}$ excess radiation attributable to free-free radiation, whereas at 2 or $3 \mu \mathrm{m}$ the contribution from this process is negligible in comparison with the stellar blackbody continuum. In deriving the law of inter- 

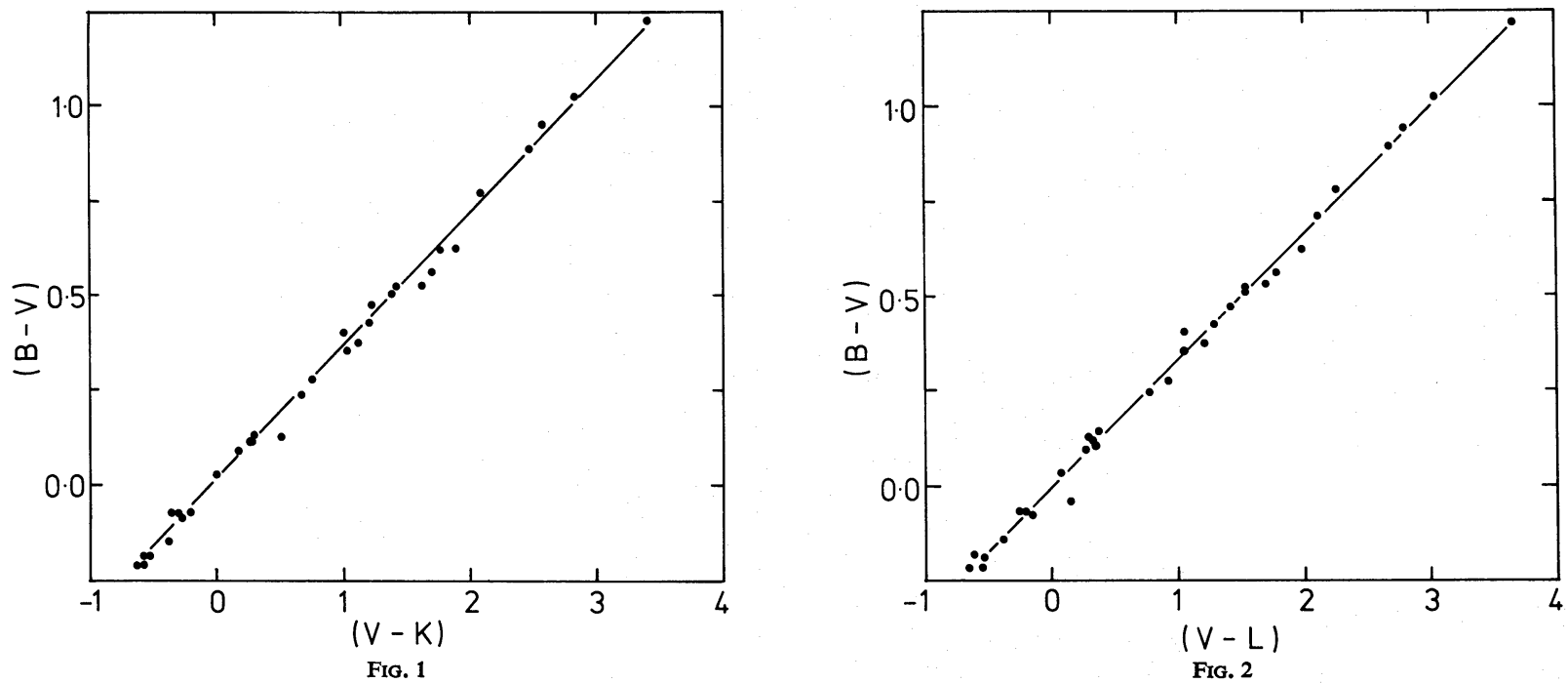

Fig. 1.- $(V-K)$ plotted versus $(B-V)$ for 33 supergiants with spectral types between 09.5 and A5. Straight line, least-squares fit to the data.

FIG. 2.-As in Fig. 1, but for $(V-L)$ versus $(B-V)$.

stellar reddening at infrared wavelengths, intrinsic colors for stars have customarily been assumed. However, the relation between intrinsic color and spectral type is not well known for early-type supergiants, primarily due to differences in the effective temperature scales for main-sequence and high-luminosity stars (e.g., Humphries, Nandy, and Kontizas 1975). The only reference for the intrinsic infrared colors of early-type supergiants (Johnson 1968 ) is unfortunately based upon too small a sample of these stars, particularly at $L$. Consequently, we have chosen here to use a method which does not depend on knowledge of the intrinsic colors but instead makes use of the observational relations (1) and (2) derived above.

The relation between the observed $L$ and $V$ magnitudes of a star is given by

$$
L=V-A_{V}-(V-L)_{0}+A_{L},
$$

where $A_{V}$ and $A_{L}$ are the total extinctions at 0.55 and $3.6 \mu \mathrm{m}$, and the subscript zero denotes the intrinsic color of the stars. From this we find

$$
L=V-R E(B-V)-(V-L)_{0}+l R E(B-V),
$$

where

$$
R=A_{V} / E(B-V) \quad \text { and } \quad l=A_{L} / A_{V} .
$$

Thus

$$
(V-L)-R(1-l)(B-V)=(V-L)_{0}-R(1-l)(B-V)_{0} .
$$

This may be compared directly with equation (2), derived directly from the observations, which gives

$$
(V-L)-2.96(B-V)=+0.04 \text {. }
$$

For any given star, its intrinsic color and the column density of interstellar dust determine the left-hand side of equation (3), whereas the right-hand side is a function only of its intrinsic color (i.e., its effective temperature), provided that $R$ and $l$ are constant in the interstellar medium. Figures 1 and 2 justify our assumption of uniform values of $R$ and $l$, since our sample of stars spans a longitude range of $\sim 210^{\circ}$. We may therefore identify $R(1-l)$ with the coefficient 2.96 in equation (4), provided that the right-hand side of equation (4) can be identified with the residuals of the intrinsic colors on the right-hand side of equation (3).

Assuming blackbody radiation, the Appendix gives relations for $(V-L)_{0}$ and $(B-V)_{0}$ in terms of the parameter $T_{4}=T_{\text {eff }} / 10^{4} \mathrm{~K}$. We may therefore evaluate $\left[(V-L)_{0}-2.96(B-V)_{0}\right]$ for a range of temperatures. Typically, this expression has the following values: $+0.02\left(T_{4}=1.5\right)$ and $+0.03\left(T_{4}=2.0\right.$ or 3.0). These theoretical predictions from a blackbody model for the stellar flux distribution correspond very well to the value of +0.04 which we 
identify from our observations with the residuals of the intrinsic colors on the right-hand side of equation (3). The identity $R(1-l)=2.96$ is thus justified on both sides of equation (3).

A similar equation to (3) can be derived for the case of the $K$ magnitudes:

$$
(V-K)-R(1-k)(B-V)=(V-K)_{0}-R(1-k)(B-V)_{0},
$$

where $k=A_{K} / A_{V}$. This can be compared with equation (1), which yields

$$
(V-K)-2.83(B-V)=-0.04 \text {. }
$$

In a procedure similar to that adopted above, we can then identify the quantity $R(1-k)$ with the value 2.83 and the residuals of the intrinsic colors on the right-hand side of equation (5) with the value -0.04 (blackbody models predict $\left[(V-K)_{0}-2.83(B-V)_{0}\right]=-0.01\left(T_{4}=1.5\right.$ or 2$)$ or $\left.-0.025\left(T_{4}=3\right)\right]$. We thus have

$$
\begin{aligned}
& R(1-l)=2.96 \pm 0.03, \\
& R(1-k)=2.83 \pm 0.04 .
\end{aligned}
$$

For reddening laws of the standard type, the quantities $l$ and $k$ are invariant for varying values of $R$; their values can be obtained, for example, from the tabulation presented by Johnson (1968) for the van de Hulst curve No. 15. This yields

$$
l \equiv A_{L} / A_{V}=4.6 \times 10^{-2} \quad \text { and } \quad k \equiv A_{K} / A_{V}=8.9 \times 10^{-2} .
$$

Thus we obtain $R=3.103 \pm 0.031$ from equation (7) and $R=3.107 \pm 0.044$ from equation (8). Combining these two results, we finally obtain $R=3.10 \pm 0.03$.

Hackwell and Gehrz (1974) have derived a value of $R=3.40 \pm 0.15$ from infrared data on supergiants with spectral types, from B1 to K5. However, this larger value of $R$ results mainly from their inclusion of data for the $10 \mu \mathrm{m}$ region. Their criterion for exclusion of a star from their reddening determination, namely [8.7] - [11.4] > 0.5 , is sufficient to exclude stars having circumstellar dust emission but does not exclude stars having even strong free-free excess emission in this region (cf. the Be stars [Gehrz, Hackwell, and Jones 1974], and Wolf-Rayet stars [Hackwell, Gehrz, and Smith 1974; Cohen, Barlow, and Kuhi 1975]). While the early-type supergiants discussed in this paper have milder excesses than do Be or Wolf-Rayet stars, nevertheless these excesses are sufficient to interfere with attempts to derive the reddening law using $10 \mu \mathrm{m}$ data.

The value of $R$ determined here is that appropriate for the diffuse interstellar clouds and intercloud medium intercepted in the path lengths toward these supergiant stars. There is no evidencs for any variation of $R$ over our observed longitude range of $\sim 210^{\circ}$ with a distance range of up to $2 \mathrm{kpc}$. As has been shown by Carrasco, Strom, and Strom (1973), larger values of $R$ may occur in the interiors of very dense shielded clouds.

The value for $R$ derived here is in good agreement with several other recent determinations of $R$ by various methods. Schalen (1975) has derived $R \approx 3.1$ from observations of dark clouds. Harris (1973) has derived $R=$ $3.15 \pm 0.20$ using the cluster diameter method; Schultz and Wiemer (1975) have derived $R=3.14 \pm 0.10$ using the color-difference method with an assortment of infrared data from various sources. The present value of $R=$ $3.10 \pm 0.03$ has the smallest error limits of any determination so far.

Finally, equations (6) and (4) may be used to derive intrinsic $(V-K)_{0}$ and $(V-L)_{0}$ colors if intrinsic $(B-V)_{0}$ colors are known.

\section{LONG WAVELENGTH INFRARED OBSERVATIONS}

The $3.6 \mu \mathrm{m}$ magnitudes of all the supergiants observed lie on the blackbody curve (§ III). Therefore, in order to assess excesses at $10 \mu \mathrm{m}$, we have used the index [3.6] - [10] (for the few cases where no $10 \mu \mathrm{m}$ magnitude was available, the $11.3 \mu \mathrm{m}$ magnitude was used). However, this color index must first be corrected for two effects: $(a)$ the effects of interstellar reddening; and $(b)$ the intrinsic color index of the star.

The reddening correction can be computed as

$$
\Delta([3.6]-[10])=A_{10}-A_{L}=-0.033 A_{V}
$$

(where $A_{10}$ is the extinction at $10 \mu \mathrm{m}$ ), since, for standard reddening laws, $A_{V}: A_{L}: A_{10}=1.00: 0.046: 0.013$. The values of $A_{V}$ were obtained by using the tabulation of $(B-V)_{0}$ versus spectral type and $M_{V}$ presented by Brunet (1975) for supergiant stars. We have taken $A_{V}=3.10 E(B-V)$ using these intrinsic colors and the $(B-V)$ colors given in Table 1.

The intrinsic infrared color index of a star can be computed by the method presented in Appendix A, provided the stellar effective temperature is known. The effective temperature scale for supergiants is not accurately known at present, but we have used the following procedure to obtain an approximate temperature scale. Humphries, Nandy, and Kontizas (1975) have fitted the ultraviolet energy distributions of several early-type supergiants with 
TABLE 2

Adopted Supergiant Temperature Scale

\begin{tabular}{|c|c|}
\hline Spectral Type & $T_{\text {eff }}(\mathrm{K})$ \\
\hline 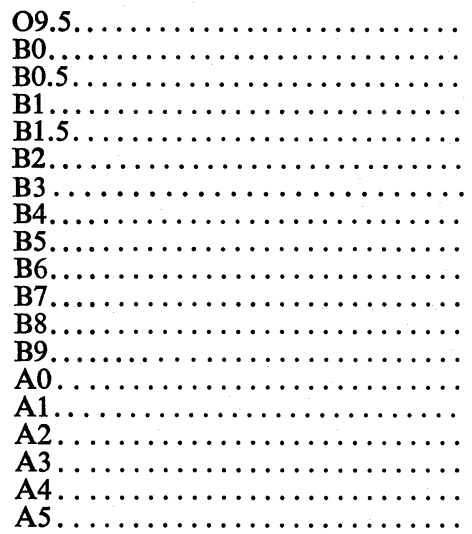 & $\begin{array}{r}30000 \\
25000 \\
22000 \\
20000 \\
19000 \\
18000 \\
15000 \\
14300 \\
13500 \\
12900 \\
12400 \\
11500 \\
10800 \\
9600 \\
9330 \\
9040 \\
8750 \\
8480 \\
8310\end{array}$ \\
\hline
\end{tabular}

equivalent blackbody energy distributions, thereby deriving approximate effective temperatures. Their data and some more recent unpublished data from the same source (Nandy 1975, private communication) make it possible to derive the ratio of supergiant to main-sequence effective temperatures for spectral types B1, B3, B5, and B8; where the main-sequence effective temperature scale of Schild, Peterson, and Oke (1971) is adopted, in conjunction with the relation between spectral type and $(B-V)_{0}$ for main-sequence stars given by Morton and Adams (1968). To obtain the ratio of supergiant to main-sequence effective temperature for intermediate spectral types, we have interpolated linearly in the range B3-B8, and for all spectral types earlier than B3 have adopted the ratio which holds for both B1 and B3. For spectral types A0 and later, the main-sequence scale of Schild, Peterson, and Oke (1971) was adopted, since the data of Humphries, Nandy, and Kontizas indicate little difference in temperature between supergiant and dwarf A stars. Table 2 presents the adopted (spectral type, effective temperature)-scale for supergiants. Errors likely to occur in this temperature scale will have only a small effect on the infrared intrinsic color corrections. The adopted temperature scale shows good agreement with the supergiant effective temperatures determined by Code et al. (1976).

Column (4) of Table 3 presents the index [3.6] - [10], where [3.6] is the $3.6 \mu \mathrm{m}$ magnitude predicted by (4) and [10] is the observed $10 \mu \mathrm{m}$ magnitude. This index is then corrected for interstellar reddening, using equation (9) ( $A_{V}$ is tabulated in column [5] and the index corrected for reddening is presented in column [6]). Finally, the index is corrected for intrinsic color by the method outlined above. Column (7) tabulates the final value of the [3.6] - [10] index corrected for both reddening and intrinsic color, which we denote as ([3.6] - [10]) excess. $_{\text {. Column (7) also }}$ includes the estimated uncertainty in the final value of $([3.6]-[10])_{\text {excess }}$, which represents the $1 \sigma$ photometric error at $10 \mu \mathrm{m}$. Column (8) of Table 3 lists the absolute visual magnitude $M_{V}$ of each star, primarily taken from Lesh (1968), Hutchings (1970), Conti and Alschuler (1971), and Rosendhal (1973). Column (9) lists the distance $D$ of each star in kpc, derived using the $M_{V}$ in column (8) in conjunction with the value of $A_{V}$ in column (5) and the $V$-magnitude in Table 1.

In Figure 3 we plot ([3.6] - [10]) excess versus spectral type for the supergiants observed. It is clear from Figure 3 that significant excesses occur for spectral types earlier than B6, whereas excesses are smaller or nonexistent for later spectral types. It should be pointed out that neither the existence of the excesses nor their pattern of occurrence with spectral type is an artifact of the corrections applied to the observed [3.6] - [10] indices, as may be seen from inspection of the data in Table 3.

It is interesting to note that a similar separation by spectral type at about B6 was found by Rosendhal (1973) in the context of the strength of $\mathrm{H} \alpha$ emission from early-type supergiants. Rosendhal interpreted this as evidence that the mechanism driving the supergiant mass loss flows changes at a stellar effective temperature of $\sim 13,000 \mathrm{~K}$ (however, see $\S$ VII). Rosendhal (1973) noticed the separation by spectral type in graphs of $\mathrm{H} \alpha$ emission strength versus $M_{V}$ and $M_{\text {bol }}$. We have plotted ([3.6] - [10]) excess versus $M_{V}$ for our supergiants and find essentially no dependence on $M_{V}$ for stars with spectral type later than B6, and a weak dependence for the sample with earlier spectral types. However, this lack of strong dependence can be understood on the grounds that, although two stars of the same spectral type may have the same ([3.6] - [10]) excess, the star with the larger luminosity can have a larger excess flux (and therefore larger mass loss rate; see $\S \mathrm{V}$ ), since the effect at $10 \mu \mathrm{m}$ of an increase in stellar luminosity can be to balance a corresponding increase in the circumstellar free-free flux at that wavelength. 
Table 3

The $10 \mu$ Excesses

\begin{tabular}{|c|c|c|c|c|c|c|c|c|}
\hline $\mathrm{HD}$ & Name & Sp. Type & {$[3.6]-[10]$} & $\mathrm{A}_{\mathrm{V}}$ & $\begin{array}{l}{[3.6]-[10]} \\
\text { reddening } \\
\text { corrected }\end{array}$ & $([3.6]-[10])$ excess & $\mathrm{M}_{\mathrm{V}}$ & $\begin{array}{c}D \\
(k p c)\end{array}$ \\
\hline 36486 & $\delta$ Ori & $09.5 \mathrm{I}$ & 0.09 & 0.22 & 0.08 & $0.19 \pm 0.20$ & -6.3 & 0.46 \\
\hline 37742 & $\zeta$ Ori & $09.5 \mathrm{I}$ & 0.02 & 0.22 & 0.01 & $0.11 \pm 0.05$ & -6.8 & 0.46 \\
\hline 37128 & $\varepsilon$ Ori & $\mathrm{BOIa}$ & 0.20 & 0.19 & 0.19 & $0.29 \pm 0.08$ & -6.8 & 0.45 \\
\hline 38771 & k Ori & $\mathrm{B} 0.5 \mathrm{Ia}$ & 0.10 & 0.19 & 0.09 & $0.18 \pm 0.16$ & -6.3 & 0.42 \\
\hline 169454 & & $\mathrm{BlIa}^{+}$ & 0.33 & 3.41 & 0.23 & $0.31 \pm 0.18$ & -7.7 & 1.48 \\
\hline 2905 & $\kappa$ Cas & $\mathrm{B} \perp \mathrm{Ia}$ & 0.19 & 0.96 & 0.16 & $0.24 \pm 0.06$ & -6.9 & 1.01 \\
\hline 24398 & $\zeta$ Per & $\mathrm{B} 1 \mathrm{Ib}$ & -0.09 & 0.99 & -0.12 & $-0.04 \pm 0.03$ & -5.6 & 0.32 \\
\hline 91316 & $\rho$ Leo & BlIab & 0.14 & 0.19 & 0.13 & $0.21 \pm 0.13$ & -6.4 & 0.97 \\
\hline 190603 & & $\mathrm{Bl} .5 \mathrm{Ia}^{+}$ & 0.43 & 2.20 & 0.36 & $0.43 \pm 0.12$ & -7.5 & 1.57 \\
\hline 194279 & & $\mathrm{~B} 1.5 \mathrm{Ia}$ & 0.38 & 3.69 & 0.26 & $0.33 \pm 0.20$ & -6.9 & 1.09 \\
\hline 14143 & & $\mathrm{~B} 2 \mathrm{Ia}$ & 0.24 & 2.02 & 0.18 & $0.25 \pm 0.30$ & -7.1 & 2.18 \\
\hline 41117 & $x^{2}$ ori & $\mathrm{B} 2 \mathrm{Ia}$ & 0.34 & 1.30 & 0.30 & $0.37 \pm 0.10$ & -7.4 & 1.38 \\
\hline 14134 & & B3Ia & 0.24 & 1.89 & 0.18 & $0.24 \pm 0.23$ & -7.0 & 2.12 \\
\hline 53138 & $\mathrm{o}^{2} \mathrm{CMa}$ & B3Ia & 0.48 & 0.19 & 0.47 & $0.53 \pm 0.10$ & -7.1 & 0.98 \\
\hline 198478 & 55 Cyg & B3Ia & 0.21 & 1.67 & 0.15 & $0.21 \pm 0.10$ & -6.8 & 0.97 \\
\hline 58350 & n $\mathrm{CM}$ & B5Ia & 0.10 & 0.11 & 0.09 & $0.13 \pm 0.08$ & -6.8 & 0.65 \\
\hline 36371 & $x$ Aur & B5Iab & 0.43 & 1.46 & 0.38 & $0.42 \pm 0.10$ & -7.1 & 1.20 \\
\hline 164353 & $67 \mathrm{Oph}$ & B5Ib & 0.04 & 0.50 & 0.03 & $0.07 \pm 0.25$ & -5.8 & 0.69 \\
\hline 15497 & & $\mathrm{~B} 6 \mathrm{Ia}$ & 0.22 & 2.76 & 0.13 & $0.17 \pm 0.17$ & -7.3 & 2.05 \\
\hline 183143 & & B7 Ia & 0.07 & 4.09 & -0.07 & $-0.04 \pm 0.10$ & -7.5 & 1.11 \\
\hline 34085 & B Ori & B8Ia & 0.04 & 0.09 & 0.04 & $0.06 \pm 0.02$ & -8.3 & 0.47 \\
\hline 166937 & $\mu \mathrm{Sgr}$ & B8Ia & 0.13 & 0.93 & 0.10 & $0.12 \pm 0.10$ & -8.1 & 1.60 \\
\hline 208501 & $13 \mathrm{Cep}$ & B8Ib & 0.02 & 2.48 & -0.06 & $-0.04 \pm 0.16$ & -6.0 & 0.74 \\
\hline 21291 & & B9Ia & 0.12 & 1.52 & 0.07 & $0.09 \pm 0.08$ & -7.1 & 0.89 \\
\hline 202850 & $\sigma$ Cyg & B9Iab & -0.09 & 0.59 & -0.11 & $-0.09 \pm 0.18$ & -6.3 & 0.95 \\
\hline 212953 & $4 \mathrm{Lac}$ & B9Iab & -0.07 & 0.50 & -0.09 & $-0.07 \pm 0.19$ & -6.0 & 1.02 \\
\hline 21389 & & AOIa & 0.28 & 1.88 & 0.21 & $0.21 \pm 0.06$ & -6.9 & 0.82 \\
\hline 87737 & $n$ Leo & $\mathrm{AOIb}$ & 0.03 & 0.06 & 0.03 & $0.03 \pm 0.08$ & -5.3 & 0.56 \\
\hline 12953 & & Al Ia & 0.24 & 1.86 & 0.17 & $0.16 \pm 0.08$ & -7.9 & 2.20 \\
\hline 14489 & 9 Per & $\mathrm{A} 2 \mathrm{Ia}$ & 0.20 & 1.21 & 0.16 & $0.15 \pm 0.12$ & -7.4 & 1.88 \\
\hline 197345 & $\alpha$ Cyg & A2 Ia & 0.15 & 0.19 & 0.14 & $0.13 \pm 0.01$ & -7.8 & 0.61 \\
\hline 207260 & $v$ Cep & $\mathrm{A} 2 \mathrm{Ia}$ & -0.17 & 1.71 & -0.23 & $-0.24 \pm 0.09$ & -6.4 & 0.63 \\
\hline 17378 & & $\mathrm{~A} 5 \mathrm{Ia}$ & 0.12 & 2.39 & 0.05 & $0.03 \pm 0.13$ & -7.8 & 2.16 \\
\hline
\end{tabular}

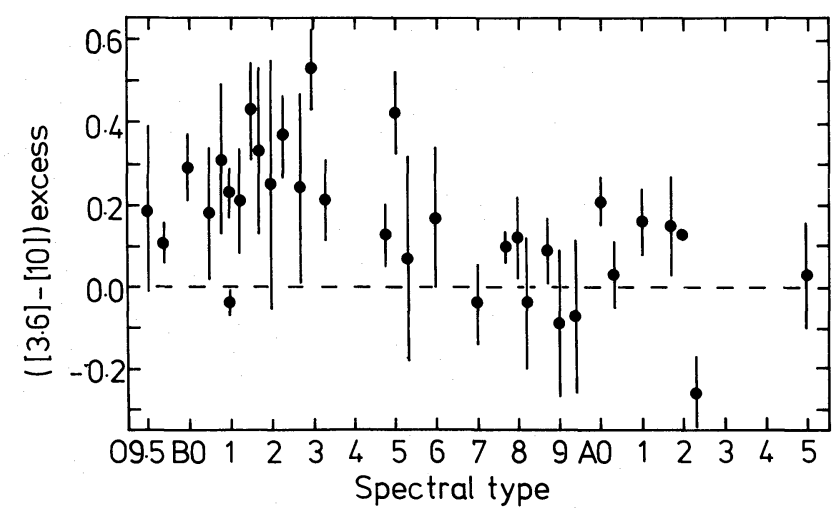

FIG. 3.-The index ([3.6] - [10]) excess, defined in the text, versus spectral type for 33 supergiants. The index is a measure of the excess at $10 \mu \mathrm{m}$ over a blackbody energy distribution. The error bars represent the $1 \sigma$ photometric errors at $10 \mu \mathrm{m}$. The horizontal dotted line corresponds to zero excess. Positive values of the index correspond to excess flux at $10 \mu \mathrm{m}$. 
The sizes of the $10 \mu \mathrm{m}$ excesses do not lend themselves to an interpretation in terms of thermal emission by dust grains. Instead, the excesses may be straightforwardly interpreted as due to free-free emission from the ionized mass loss outflows from these stars. In the next section, we consider in detail the origin of the excesses.

\section{THE VELOCITY LAW FOR P CYGNI AND MASS LOSS RATES FOR EARLY-TYPE SUPERGIANTS}

As shown by Wright and Barlow (1975) and Panagia and Felli (1975), constant-velocity ionized mass loss outflows, which give rise to an $R^{-2}$ density distribution, generate a $\nu^{2 / 3}$ free-free emission spectrum. The observed freefree flux from a star at a given frequency can thus be used to determine the mass loss rate under the assumption that the flux observed at that frequency originates from the constant-velocity region of the outflow.

The free-free flux at $10 \mu \mathrm{m}$ was determined for each star as follows. The intrinsic (blackbody) $10 \mu \mathrm{m}$ magnitude of a star, $[10]_{0}$, is given by

$$
[10]_{0}=[10]_{\mathrm{obs}}-A_{10}+([3.6]-[10])_{\text {excess }} \text {, }
$$

where [10] $]_{\mathrm{obs}}$ is the observed $10 \mu \mathrm{m}$ magnitude and $A_{10}=0.013 A_{V}$ is the extinction at $10 \mu \mathrm{m}$. The observed $10 \mu \mathrm{m}$ magnitude corrected for extinction, $[10]_{\text {corr }}$, is given by

$$
[10]_{\mathrm{corr}}=[10]_{\mathrm{obs}}-A_{10} \text {. }
$$

The free-free flux is then the difference between the fluxes corresponding to $[10]_{\text {corr }}$ and $[10]_{0}$. Column (4) of Table 4 presents, for each star, the $10 \mu \mathrm{m}$ free-free flux $S_{v}(10 \mu)$ (in janskys). For three stars not having a $10 \mu \mathrm{m}$ magnitude, the $11.3 \mu \mathrm{m}$ magnitude was used to obtain instead the excess flux at $11.3 \mu \mathrm{m}$. Six of the supergiants have $10 \mu \mathrm{m}$ magnitudes fainter than the theoretically predicted blackbody magnitudes. For four of these stars, upper limits to the $10 \mu \mathrm{m}$ free-free excess have been obtained instead, by using the upper limit to the $10 \mu \mathrm{m}$ magnitude defined by the $1 \sigma$ photometric errors. For two stars, however ( $\zeta$ Per and $\nu$ Cep), even the $1 \sigma$ photometric errors do not take the $10 \mu \mathrm{m}$ magnitudes above the blackbody level, and so no upper limit is quoted for the $10 \mu \mathrm{m}$ excess of these stars.

Wright and Barlow (1975) have derived the following formula for the mass loss rate $\dot{M}$ of a star undergoing uniform velocity mass outflow:

$$
\dot{M}=\frac{0.095 \mu v_{\infty} S_{\nu}^{3 / 4} D^{3 / 2}}{Z \gamma^{1 / 2} g^{1 / 2} \nu^{1 / 2}} M_{\odot} \mathrm{yr}^{-1},
$$

where $v_{\infty}$ is the terminal velocity of the mass outflow in $\mathrm{km} \mathrm{s}^{-1}, S_{v}$ is the free-free flux (in Jy) observed at a frequency $\nu, D$ is the distance of the star in kpc, $\mu$ is the mean atomic weight of the gas, $Z$ is the mean ionic charge of the gas, $\gamma$ is the mean number of electrons per ion, and $g(\nu, T)$ is the Gaunt factor. For the present case, $\mu=$ $1.26, Z=1.0$, and $\gamma=1.0$. Values of the Gaunt factor $g(\nu, T)$ can be obtained from Karzas and Latter (1961); the assumption was made that the temperature of the wind was the same as the effective temperature of the star.

However, the mass loss rates derived using equation (12) (these mass loss rates are not tabulated) are found to considerably exceed (by factors of up to 5 for the earliest supergiants) the upper limit for mass loss by radiation pressure, which is equal to $L / v_{\infty} c$ (Cassinelli and Castor 1973), where $L$ is the luminosity of the star and $c$ is the velocity of light. Further, if the characteristic radius from which the $10 \mu \mathrm{m}$ radiation originates is calculated using the formula of Wright and Barlow (1975),

$$
R(\nu)=2.8 \times 10^{28} \gamma^{1 / 3} g^{1 / 3} Z^{2 / 3} T^{-1 / 2}\left(\dot{M} / \mu v_{\infty} \nu\right)^{2 / 3} \mathrm{~cm},
$$

then it is found that the characteristic radii derived are less than the photospheric radii $\boldsymbol{R}_{c}$ by factors of up to 5 for the earliest supergiants. This indicates a breakdown of the simple uniform-flow model. Three possible reasons suggest themselves for these discrepant results. (a) The wind temperature may be very much higher than the effective temperature of the star. (b) The wind has strong density fluctuations giving rise to a clumping effect. $(c)$ The $10 \mu \mathrm{m}$ radiation originates from the region where the stellar wind is still undergoing acceleration, rendering the constantvelocity model inapplicable. We consider each of these possibilities in turn.

a) In the constant-velocity model, there is no dependence of the flux $S_{v}$ upon the temperature of the gas in the wind, except extremely weakly through the Gaunt factor (see eq. [12]). The physical reason for this nondependence upon temperature is outlined by Castor, Abbott, and Klein (1976). Further, since the characteristic radius $R(\nu)$ is proportional to $T^{-1 / 2}$, a higher wind temperature only exacerbates the discrepancies of the uniform-flow model.

b) Consider a constant-velocity stellar wind which experiences density fluctuations giving rise to clumps in which the density is increased by a factor of $f$ over the uniform value, with no gas between the clumps. It is then an easy matter to show, following the same analysis as given by Wright and Barlow (1975), that the flux $S_{v}$ emitted at a given frequency will be increased by a factor of $f^{4 / 9}$ over the uniform-flow value. Thus if equation (12) is used to 
obtain $\dot{M}$, the mass loss rates will be overestimated by a factor of $f^{1 / 3}$. The characteristic radius $R(\nu)$ will be increased by a factor of $f^{5 / 9}$ in the clumping model, so that the values of $f$ which are required to reduce the mass loss rates below the upper limit for radiation pressure $(f \approx 10-50)$ will also increase the characteristic $10 \mu \mathrm{m}$ radii to values greater than the photospheric radii $R_{c}$. Density clumping might thus appear to offer an explanation for the discrepancies of the simple constant-velocity uniform-flow model. However, two lines of evidence may be used to show that density clumping is not the factor responsible.

1. Castor, Abbott, and Klein (1976) and Conti and Frost (1977) have derived mass loss rates for several earlytype stars by applying the mass loss theory of Castor, Abbott, and Klein (1975) to observed H $\alpha$ fluxes. Since that theory is equivalent to the constant-velocity model in its effects, their results may be compared with the results from the infrared constant-velocity model in order to check for consistency. Since the $\mathrm{H} \alpha$ emission is optically thin in the models of Castor, Abbott, and Klein, density clumping is found to lead to an increase in the $\mathrm{H} \alpha$ flux by a factor of $f$ over the uniform case, and mass loss rates will be overestimated by a factor of $f^{1 / 2}$. Thus if clumping actually takes place, the $\mathrm{H} \alpha$ fluxes should yield mass loss rates which are larger than those derived from infrared fluxes. The present paper and those of Castor, Abbott, and Klein (1976) and Conti and Frost (1977) have three stars in common: $\zeta$ Ori, HD 14947, and $\zeta$ Pup. The uniform-flow model applied to the $10 \mu$ m excess flux of $\zeta$ Ori yields a mass loss rate of $\dot{M}=8.2 \times 10^{-6} M_{\odot} \mathrm{yr}^{-1}$, whereas Castor, Abbott, and Klein obtain $\dot{M}=3.2 \times 10^{-6} M_{\odot} \mathrm{yr}^{-1}$ from the $\mathrm{H} \alpha$ flux. Similarly, the uniform-flow model applied to the $3.6 \mu \mathrm{m}$ excess flux of HD 14947 (§ VI) yields $\dot{M}=1.4 \times 10^{-5} M_{\odot} \mathrm{yr}^{-1}$, whereas Conti and Frost obtain $\dot{M}=8.1 \times 10^{-6} M_{\odot} \mathrm{yr}^{-1}$ from the $\mathrm{H} \alpha$ flux. Finally, the $2.2 \mu \mathrm{m}$ excess flux of $\zeta$ Pup ( $(\mathrm{VI})$ yields $\dot{M}=2.1 \times 10^{-5} M_{\odot} \mathrm{yr}^{-1}$, whereas Conti and Frost obtain $\dot{M}=$ $8.9 \times 10^{-6} M_{\odot} \mathrm{yr}^{-1}$ from the $\mathrm{H} \alpha$ flux. Thus in all cases the $\mathrm{H} \alpha$ fluxes yield mass loss rates less than those derived from infrared fluxes, in disagreement with the predictions of a density-clumping model.

2. Although density clumping increases the flux emitted from a constant-velocity wind, it does not alter the spectral index of the emitted free-free radiation. The high-luminosity B1 supergiant P Cygni has been observed at both radio and infrared frequencies (Wendker, Baars, and Altenhoff 1973; Gehrz, Hackwell, and Jones 1974; cf. Wright and Barlow 1975). Between $10 \mu \mathrm{m}$ and $10.7 \mathrm{GHz}$, the spectral index of the excess radiation is 0.75 , compared to a spectral index of 0.56 (taking into account the Gaunt factor) predicted by the constant-velocity model (with or without clumping). The spectral index discrepancy cannot be explained by a constant-velocity model but can be explained by an accelerating flow model.

c) Neglecting the frequency dependence of the Gaunt factor, we find that a density distribution $n \propto R^{-\beta}$, where $R$ is the radius, gives rise to a spectrum $S_{v} \propto \nu^{(4 \beta-6 / 2 \beta-1)}$ (Wright and Barlow 1975). Constant-velocity outflow, corresponding to $\beta=2$, gives $S_{v} \propto \nu^{2 / 3}$. An accelerating outflow will give $\beta>2$, and a steeper spectrum will result. Therefore an accelerating flow model can explain the steep spectral energy distribution of $\mathbf{P}$ Cyg and reduce the mass loss rates derived from the infrared fluxes. In the remainder of this section, we use the radio and infrared data for P Cyg to deduce the velocity law; by applying this velocity law, we then derive mass loss rates for the other supergiants.

If the uniform-flow theory is applied to the flux of $15 \mathrm{mJy}$ at $10.68 \mathrm{GHz}$ detected by Wendker, Baars, and Altenhoff (1973), then $\dot{M} / v_{\infty}=5 \times 10^{-8}\left(M_{\odot} \mathrm{yr}^{-1}\right) /\left(\mathrm{km} \mathrm{s}^{-1}\right)$ is derived, using equation (12). Using equation (13), this leads to $R(10 \mathrm{GHz})=1.3 \times 10^{4} R_{\odot}=147 R_{c}$, where $R_{c}=89.2 R_{\odot}$ for $\mathrm{P}$ Cyg $\left[M_{\mathrm{bol}}(\mathrm{P} \mathrm{Cyg})=-10.4\right.$; $\left.T_{\text {eff }}(\mathrm{P} \mathrm{Cyg})=2 \times 10^{4} \mathrm{~K}\right]$. For any physically reasonable acceleration law, one can be reasonably certain that the flow will have attained the terminal velocity $v_{\infty}$ at such a large radius, and the spectral index of 0.65 between 2.8 and $6 \mathrm{~cm}$ is consistent with emission from a constant-velocity region. The radio data therefore directly yield $\dot{M} / v_{\infty}$ for P Cyg, and this value of $\dot{M} / v_{\infty}$ may then be used to deduce the velocity law in the infrared emitting region of the mass outflow.

Wright and Barlow (1975) obtained the expression for the emergent free-free flux analytically for the case of a constant-velocity outflow, but for velocity laws more complicated than this, an analytic solution cannot in general be obtained. Therefore use was made of a generalized spherical radiative transfer program originated by Kunasz and Kunasz (1977), and of a spherical transfer program due to P. B. Kunasz which incorporated electron-scattering and used the variable Eddington-factor method described by Kunasz and Hummer (1974). This program was used to calculate the emergent flux spectrum from an opaque stellar core surrounded by an expanding wind with any given velocity law. In the form used, the stellar core of photospheric radius $R_{c}$ radiated as a Planckian blackbody of effective temperature $T$. The temperature of the gas in the wind was set equal to the effective temperature of the star. The alternative case of a much higher wind temperature is considered below.

The infrared free-free spectrum of P Cyg was obtained from the photometry of Gehrz, Hackwell, and Jones (1974), taking $V=4.80,(B-V)=0.40$ (Fernie 1969) and using the method described earlier to deduce the excess flux at each of the observed wavelengths. An intrinsic color of $(B-V)_{0}=-0.16$ was taken from Brunet (1975), yielding $A_{V}=1.74$. The excess flux at each wavelength observed is plotted in Figure 4 . The aim of the model calculations was to find a velocity law which gave a fit to the ten flux density data points between $\nu=1.3 \times$ $10^{14} \mathrm{~Hz}$ and $5 \times 10^{9} \mathrm{~Hz}$.

The fundamental parameters needed to calculate the emergent infrared and radio spectrum are: the stellar radius $R_{c}$, the stellar effective temperature (and the wind temperature) $T$, the mass loss rate divided by the terminal velocity, $\dot{M} / v_{\infty}$, the distance of the star $D$, and the velocity law $v(R)$. For P Cyg we have $R_{c}=89.2 R_{\odot}, T=2 \times 10^{4} \mathrm{~K}$, $\dot{M} / v_{\infty}=5 \times 10^{-8}\left(M_{\odot} \mathrm{yr}^{-1}\right) /\left(\mathrm{km} \mathrm{s}^{-1}\right)$, and $D=1.8 \mathrm{kpc}\left(M_{V}=-8.4 ;\right.$ cf. Hutchings $\left.1976 a\right)$. 


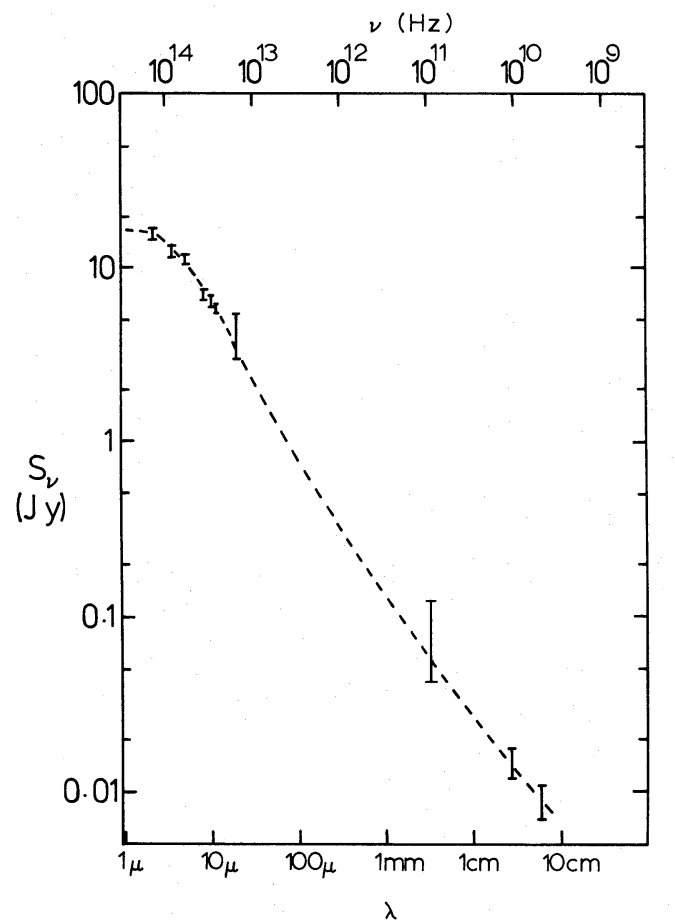

FIG. 4.-Flux density $S_{v}$ versus frequency $v$ and wavelength $\lambda$ for $\mathrm{P}$ Cyg. The error bars show the observed fluxes. The radio fluxes are from Wendker et al. 1973, and the infrared fluxes were derived from the photometry of Gehrz et al. 1974 by the method described in the text. The $3.3 \mathrm{~mm}$ flux is from Schwartz and Spencer (1977). The dotted line shows the fit given by a mass loss model having a velocity law given by equation (20) and a mass loss rate divided by terminal velocity of $\dot{M} / v_{\infty}=5 \times 10^{-8}\left(M_{\odot} \mathrm{yr}^{-1}\right)$ $\left(\mathrm{km} \mathrm{s}^{-1}\right)^{-1}$.

The first velocity law which was tried was the usual radiative acceleration law (e.g., similar to that found by Castor, Abbott, and Klein 1975). This law is of the form

$$
v(R)=v_{\infty}\left[1-\left(1-X_{\mathrm{c}}\right) \frac{R_{c}}{R}\right]^{1 / 2} .
$$

Here $X_{c}=\left(v_{c} / v_{\infty}\right)^{2}$, where $v_{c}$ is the velocity of the flow at $R_{c}$. The value of $X_{c}$ for P Cyg was found as follows. For the velocity law (14) the value of the photospheric density is given by

$$
n_{c}=\frac{\dot{M}}{4 \pi \mu m_{\mathrm{H}} v_{\infty} R_{c}{ }^{2} X_{c}{ }^{1 / 2}} .
$$

Non-LTE plane-parallel model atmosphere calculations by Mihalas (1972) give good agreement with the photospheric absorption-line spectra of OB stars. For the parameters corresponding to P Cyg $\left(T=2 \times 10^{4} \mathrm{~K}, \log g=\right.$ $2.4-2.5 \mathrm{~cm} \mathrm{~s}^{-2}$ ), the tabulation of Mihalas gives $n_{c}=3 \times 10^{13} \mathrm{~cm}^{-3}$, which in conjunction with equation (15) gives $X_{c}=10^{-6}$. The velocity law (14) is plotted in Figure 5 .

It was found that a velocity law of the form (14) gives no extra flux at any frequency over that produced by a constant-velocity model. The reason for this is that in equation (14) the acceleration to $v_{\infty}$ takes place in such a short distance (see Fig. 5) that negligible optical depth is contained in the accelerating region. Since the observed $10 \mu \mathrm{m}$ free-free flux from P Cyg is 5 times that predicted by the constant-velocity model, it is obvious that the acceleration to $v_{\infty}$ must take place over a more extended region than is the case for (14). A variety of velocity laws were experimented with; each, however, had to satisfy the conditions

$$
V\left(R_{c}\right)=v_{\infty} X_{c}^{1 / 2}, \quad v(\infty)=v_{\infty} .
$$

The radio spectrum gave the additional condition that $v\left(150 R_{c}\right) \approx v_{\infty}$. It was found that a velocity law of the form

$$
v(R)=v_{\infty}\left[1-\left(1-X_{c}\right)\left(\frac{R_{c}}{R}\right)^{k}\right]^{1 / 2}
$$




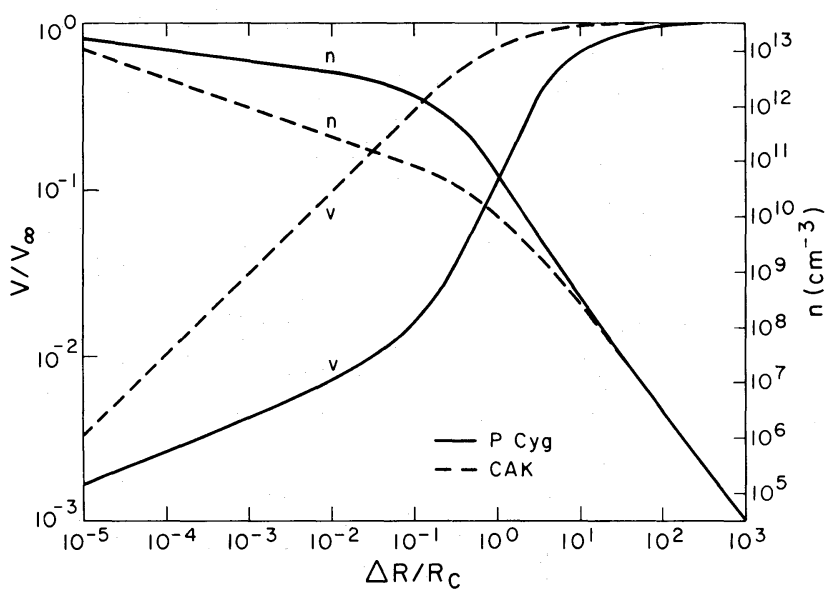

FIG. 5.-The solid lines show the velocity and density distributions produced in the atmosphere of P Cyg by the velocity law (20), as a function of incremental radius $\Delta R / R_{c}$, where $R_{c}$ is the photospheric radius and $\Delta R$ is the distance from the photosphere. For comparison, the dotted lines labeled "CAK" show the velocity and density distributions produced by the standard radiative acceleration velocity law (14), which corresponds closely to the velocity law obtained in the original models of Castor et al. (1975).

could achieve the high flux levels required in the infrared only by giving too flat a spectrum between the infrared and radio, with too much resultant radio flux. A velocity law of the form

$$
v(R)=v_{\infty} X_{c}^{0.5\left(R_{c} / R\right)^{m}}
$$

could give the correct spectrum between the radio and infrared but would then give too steep a spectrum in the infrared. A composite velocity law was found to be necessary, of the form

$$
v(R)=v_{\infty}\left[1-\left(1-X_{\mathrm{c}}\right)\left(\frac{R_{\mathrm{c}}}{R}\right)^{k}\right]^{m} X_{c}^{n\left(R_{\mathrm{c}} / R\right)^{P}},
$$

where $m+n=\frac{1}{2}$. The best fit to the observed spectrum was achieved with $k=1, m=0.21, n=0.29$, and $p=1$. The generalized form of this velocity law, in a form such that there is no dependence of the emitted flux spectrum upon $X_{c}$, is

$$
v(R)=v_{\infty} \operatorname{dex}\left[-1.74 R_{c} / R\right]\left[1-\left(1-1.91 \times 10^{8} X_{c}^{2.38}\right) \frac{R_{c}}{R}\right]^{0.21} .
$$

The spectrum generated by this velocity law is shown for P Cyg by the dotted line in Figure 4. It is found that the 10 radio, millimeter, and infrared flux data points determine the velocity law fairly precisely. The velocity and density given by equation (20) for P Cyg are plotted in Figure 5 and compared with the same quantities given by the standard radiative acceleration law (14). It should be noted that the 2.3-19.5 $\mu \mathrm{m}$ data for P Cyg define the velocity only as far out as $R \approx 4 R_{c}$. Beyond this point the velocity law may approach $v_{\infty}$ more quickly than for equation (20), without the emitted flux spectrum being significantly altered.

A velocity law similar to (14) was obtained by Castor, Abbott, and Klein (1975) in their radiation pressure mass loss theory; but more detailed models taking into account many more ions and lines have led to a more extended acceleration law (Abbott 1976, private communication), though not as yet as extended as derived here for P Cyg. Kuan and Kuhi (1975), from an analysis of hydrogen line profiles, concluded that P Cyg possessed a decelerating atmosphere with a velocity law of the form $v(R)=v_{c}\left(R_{c} / R\right)^{1 / 2}$. However, such a velocity law gives rise to a free-free spectrum with $S_{v} \propto \log (1 / v)$, corresponding to increasing flux with increasing wavelength, and is therefore ruled out by the observed free-free spectrum of P Cyg.

Rogerson and Lamers (1975) have suggested that the mass loss outflow from the B0 V star $\tau$ Sco has a temperature of $\sim 2 \times 10^{5} \mathrm{~K}$. A similar temperature for the mass loss outflow from $\zeta$ Pup has been derived by Lamers and Morton (1976). Mass loss models for P Cyg with wind temperatures of this order were experimented with, but no velocity law could be found which gave a fit to the radio and infrared data. The major effect of the higher wind temperature was to make the free-free emission optically thin at most infrared wavelengths (since free-free opacity is proportional to $T^{-1 / 2}$ ), leading to too flat an infrared spectrum. In particular, the possible alternative "hot" velocity law (21) for $\zeta$ Pup ( $(\mathrm{VI})$ does not give a fit to the observed infrared spectrum of P Cyg. The observed emission lines in the optical spectrum of P Cyg are all of low excitation, indicating that it does not possess a high wind temperature. 
Having obtained the form of the velocity law for P Cygni, we can then apply the law to the other supergiants in our sample. The assumption that the P Cygni velocity law also applies to the late B-and early A-type supergiants may be questionable, although the application gives consistent results (see § VII). It would therefore be desirable to obtain a radio detection of a later-type supergiant so that an independent derivation of the velocity law can be carried out. Of our sample, $\beta$ Ori and $\alpha$ Cyg are the most likely to be detectable (see below).

If the free-free flux $S_{\nu}$ at a frequency $\nu$ can be measured, then, given a knowledge of $R_{c}, T$, and $D$ for the particular star, the quantity $\dot{M} / v_{\infty}$ can be determined for a known velocity law, which in this case is given by (20). Columns (4), (5), and (6) of Table 4 give the values of $S_{v}(10 \mu \mathrm{m}), M_{\text {bol }}$, and $R_{c}$ for each supergiant; column (7) gives the value of the Gaunt factor $g(\nu, T)$ appropriate for each star at $10 \mu \mathrm{m} . M_{\mathrm{bol}}$ and the photospheric radius of each star were derived in exactly the same manner as described by Snow and Morton (1976), using the temperature scale of Table 2 (note that $\zeta$ Ori is a multiple system with total $M_{V}=-6.8$, with the supergiant $\zeta$ Ori A having $M_{V}=-6.4$; Snow and Morton 1976).

Column (8) of Table 4 lists the value of $\dot{M} / v_{\infty}$ determined for each star. Values of the terminal wind velocity for 10 of the present supergiants are available from the stellar wind velocity survey of Snow and Morton (1976). These are tabulated in column (9) of Table 4 and correspond to the typical velocity of the short wavelength edges of the P Cygni-type absorption components of ultraviolet lines. For the remainder of the stars, we have interpolated the value of $v_{\infty}$, and these interpolated velocities are given in parentheses in column (9). Column (10) of Table 4 lists the mass loss rates $\dot{M}$ derived using these values of $v_{\infty}$.

We have listed two values of $v_{\infty}$ for each of the supergiants $\delta, \zeta, \epsilon$, and $\kappa$ Ori. The first (and largest) value in each case corresponds to the terminal velocity derived by Snow and Morton from the N v $1240 \AA$ system. It is found if these velocities are used then the values of $\dot{M} /\left(L / v_{\infty} c\right)$, the mass loss rate divided by the upper limit for mass loss by radiation pressure, are equal to $0.51,0.45,0.95$, and 1.10 , respectively. This contrasts with the results for all the other supergiants, for which the radio is significantly less than unity (§VII). This discrepancy may be due to the velocities derived from N v being too high, since Snow and Morton comment that this particular group of stars appear to have an extended wing of $\mathrm{N} \mathrm{v}$ which may be due to some other absorption. If the terminal velocities derived from the rest of the ultraviolet lines are used instead, then the values of $\dot{M} /\left(L / v_{\infty} c\right)$ become less than unity in each case (this quantity is very sensitive to changes in $v_{\infty}$, since it is proportional to $v_{\infty}^{2}$ ). These velocities are the second of the terminal velocities listed for the four stars.

Using the lower of the terminal velocities, we can compare the derived values of $\dot{M}=0.92,1.0$, and $1.7 \times$ $10^{-6} M_{\odot} \mathrm{yr}^{-1}$, for $\delta, \zeta$, and $\epsilon$ respectively, with the values of $\dot{M}=1.0,1.2$, and $1.7 \times 10^{-6} M_{\odot} \mathrm{yr}^{-1}$ determined for the same stars respectively from rocket ultraviolet spectra by Morton (1967). Hearn (1975) has derived $\dot{M}=$ $1.8 \times 10^{-6} M_{\odot} \mathrm{yr}^{-1}$ from an analysis of the $\mathrm{H} \alpha$ line of $\zeta$ Ori, in good agreement with the present determination for this star.

The data for $\beta$ Ori present an extremely discrepant picture. As already mentioned in $\S$ III, the $U B V$ data for this star cover a range of $0.22 \mathrm{mag}$ at $V$. It was thus impossible to derive predicted 2.2 and $3.6 \mu \mathrm{m}$ magnitudes; instead, it was assumed that there was no excess at these wavelengths, as found for the other late-type supergiants. The $10 \mu \mathrm{m}$ excess found here is smaller than that found using the data of Gehrz, Hackwell, and Jones (1974), whereas the $18 \mu \mathrm{m}$ excess found by us is larger than the $19.5 \mu \mathrm{m}$ excess from Gehrz, Hackwell, and Jones. In fact, model calculations show that our $10 \mu \mathrm{m}$ excess is consistent only with the $19.5 \mu \mathrm{m}$ excess of Gehrz, Hackwell, and Jones, whereas the $10 \mu \mathrm{m}$ excess from Gehrz, Hackwell, and Jones is consistent only with our $18 \mu \mathrm{m}$ excess. In addition to these complications, Morrison and Simon (1973) found a $20 \mu \mathrm{m}$ excess significantly larger than that found by either us or Gehrz, Hackwell, and Jones. Two values of $\dot{M} / v_{\infty}$ are listed for $\beta$ Ori in Table 4 , the first from our $10 \mu \mathrm{m}$ data and the second from the $10 \mu \mathrm{m}$ data of Gehrz, Hackwell, and Jones. These values of $\dot{M} / v_{\infty}$ predict a $10 \mathrm{GHz}$ flux of between 2.2 and $4.1 \mathrm{mJy}$ from $\beta$ Ori. Altenhoff et al. (1976) quote an upper limit of $5 \mathrm{mJy}$ at 10.69 $\mathrm{GHz}$. In summary, the optical and infrared data for $\beta$ Ori indicate that it may be strongly variable. Simultaneous $U B V$ and infrared photometry of this star would be useful.

According to our photometry, $\alpha$ Cyg and $\epsilon$ Ori possess significant excesses at $18 \mu \mathrm{m}$. The $10 \mu \mathrm{m}$ excess flux of $\alpha$ Cyg predicts an excess of $2.1 \mathrm{Jy}$ at $18 \mu \mathrm{m}$, compared with an observed excess of $5.7 \pm 2.4 \mathrm{Jy}$. The $18 \mu \mathrm{m}$ excess of $\epsilon$ Ori, if real (two independent detections were made), is too large (4.6 Jy) to be explained by a free-free model and may require the presence of dust emission. However, it is possible that a blue leak existed in the $18 \mu \mathrm{m}$ filter at the time of these observations (Gehrz and Hackwell 1975, private communication), so that reobservation of these two stars at $18 \mu \mathrm{m}$ would be desirable.

At the foot of Table 4 we give the mass loss rate determinations for P Cyg and $\zeta^{1}$ Sco, two supergiants of similar characteristics. The determination quoted for P Cyg is from the $10 \mu \mathrm{m}$ flux but, as noted earlier, the velocity law was derived using this star, so all infrared and radio fluxes for P Cyg give the same mass loss rate. The terminal velocity for P Cyg is taken from Hutchings (1968). The two mass loss rate determinations listed for $\zeta^{1}$ Sco are from the $H(1.65 \mu \mathrm{m})$ and $K(2.2 \mu \mathrm{m})$ fluxes measured by Schild, Neugebauer, and Westphal (1971). Besides the parameters listed in Table 4, other relevant parameters for this star are $V=4.72,(B-V)=0.48, A_{V}=1.98$, $M_{V}=-8.2, D=1.54 \mathrm{kpc}$, and $(H-K)_{0}=-0.07$, along with the observed magnitudes of $H=3.30$ and $K=3.11$. The terminal velocity for $\zeta^{1}$ Sco is taken from Hutchings (1976b). It can be seen that the mass loss rates derived from the $H$ and $K$ fluxes of $\zeta^{1}$ Sco are in good agreement with each other. A $10 \mathrm{GHz}$ flux from $\zeta^{1}$ Sco of $10 \mathrm{mJy}$ is predicted, using equation (18) of Wright and Barlow (1975). The only other member of our sample of 
Table 4

Supergiant Mass Loss Rates

\begin{tabular}{|c|c|c|c|c|c|c|c|c|c|c|c|}
\hline HD & Name & Sp. Type & $\mathrm{s}_{v}(10 \mu)$ & Mol & $R_{c} / R_{\rho}$ & $g(\nu, T)$ & $\begin{array}{c}\dot{\mathrm{M}} / \mathrm{v}_{\infty} \\
\left(\mathrm{M}_{\Theta} / \mathrm{yr}\right) / \\
\left(\mathrm{km} \mathrm{s}^{-1}\right)\end{array}$ & $\left(k \mathrm{~m}^{\mathrm{s}^{-1}}\right)$ & $\begin{array}{c}\dot{\mathrm{M}} \\
\left(\mathrm{M}_{\Theta} / \mathrm{yr}\right)\end{array}$ & $\dot{\mathrm{M}} /\left(\mathrm{L} / \mathrm{v}_{\infty} \mathrm{c}\right)$ & $\tau_{\mathrm{e}}$ \\
\hline 36486 & $\delta$ Ori & $09.5 \mathrm{I}$ & $0.41^{*}$ & -9.2 & 22.8 & 1.8 & $3.41 \times 10^{-10}$ & $\left\{\begin{array}{l}3390 \\
2700\end{array}\right.$ & $\begin{array}{l}1.2 \times 10^{-6} \\
9.2 \times 10^{-7}\end{array}$ & $\left.\begin{array}{l}0.51 \\
0.32\end{array}\right\}$ & 0.07 \\
\hline 37742 & $\zeta$ Ori & $09.5 \mathrm{I}$ & 0.50 & -9.3 & 23.9 & 1.8 & $3.80 \times 10^{-10}$ & $\left\{\begin{array}{l}3150 \\
2700\end{array}\right.$ & $\begin{array}{l}1.2 \times 10^{-6} \\
1.0 \times 10^{-6}\end{array}$ & $\left.\begin{array}{l}0.45 \\
0.33\end{array}\right\}$ & 0.08 \\
\hline 37128 & $\varepsilon$ ori & BOIa & $1.16^{*}$ & -9.3 & 34.4 & 1.75 & $7.23 \times 10^{-10}$ & $\left\{\begin{array}{l}3320 \\
2300\end{array}\right.$ & $\begin{array}{l}2.4 \times 10^{-6} \\
1.7 \times 10^{-6}-6\end{array}$ & $\left.\begin{array}{l}0.95 \\
0.45\end{array}\right\}$ & 0.10 \\
\hline 38771 & k Ori & $\mathrm{B} 0.5 \mathrm{Ia}$ & $0.51^{*}$ & -8.5 & 30.7 & 1.7 & $3.71 \times 10^{-10}$ & $\left\{\begin{array}{l}3450 \\
1900\end{array}\right.$ & $\begin{array}{l}1.3 \times 10^{-6} \\
7.0 \times 10^{-7}\end{array}$ & $\begin{array}{l}1.10\} \\
0.33\}\end{array}$ & 0.06 \\
\hline 169454 & & $B 1 \mathrm{Ia}^{+}$ & 0.46 & -9.7 & 64.6 & 1.7 & $2.14 \times 10^{-9}$ & $(1500)$ & $3.2 \times 10^{-6}$ & 0.40 & 0.16 \\
\hline 2905 & א Cas & B1Ia & 0.32 & -8.9 & 44.7 & 1.7 & $9.04 \times 10^{-10}$ & $(1500)$ & $1.4 \times 10^{-6}$ & 0.35 & 0.10 \\
\hline 91316 & $\rho$ Leo & B1Iab & 0.18 & -8.3 & 31.0 & 1.7 & $5.46 \times 10^{-10}$ & 1580 & $8.6 \times 10^{-7}$ & 0.40 & 0.09 \\
\hline 190603 & & $\mathrm{~B} 1.5 \mathrm{Ia}^{+}$ & 0.55 & -9.4 & 62.4 & 1.7 & $2.85 \times 10^{-9}$ & $(1000)$ & $2.9 \times 10^{-6}$ & 0.31 & 0.22 \\
\hline 194279 & & $\mathrm{~B} 1.5 \mathrm{Ia}$ & 0.44 & -8.8 & 47.3 & 1.7 & $1.30 \times 10^{-9}$ & $(1000)$ & $1.3 \times 10^{-6}$ & 0.24 & 0.13 \\
\hline 14143 & & $\mathrm{~B} 2 \mathrm{Ia}$ & 0.10 & -8.9 & 55.2 & 1.65 & $1.23 \times 10^{-9}$ & $(1000)$ & $1.2 \times 10^{-6}$ & 0.21 & 0.11 \\
\hline 41117 & $x^{2}$ ori & $\mathrm{B} 2 \mathrm{Ia}$ & 0.53 & -9.2 & 63.4 & 1.65 & $2.22 \times 10^{-9}$ & $(1000)$ & $2.2 \times 10^{-6}$ & 0.28 & 0.17 \\
\hline 14134 & & B3Ia & 0.10 & -8.35 & 61.7 & 1.55 & $1.22 \times 10^{-9}$ & $(580)$ & $7.1 \times 10^{-7}$ & 0.12 & 0.10 \\
\hline 53138 & $\mathrm{o}^{2} \mathrm{CMa}$ & B3Ia & 1.32 & -8.45 & 64.6 & 1.55 & $2.90 \times 10^{-9}$ & 580 & $1.7 \times 10^{-6}$ & 0.25 & 0.22 \\
\hline 198478 & 55 Cyg & B3Ia & 0.34 & -8.15 & 56.3 & 1.55 & $9.58 \times 10^{-10}$ & $(580)$ & $5.6 \times 10^{-7}$ & 0.11 & 0.08 \\
\hline 58350 & n $\mathrm{CMa}$ & $\mathrm{B} 5 \mathrm{Ia}$ & 0.46 & -7.8 & 59.1 & 1.5 & $7.15 \times 10^{-10}$ & 590 & $4.2 \times 10^{-7}$ & 0.12 & 0.06 \\
\hline 36371 & $x$ Aur & B5Iab & 0.70 & -8.1 & 67.9 & 1.5 & $2.39 \times 10^{-9}$ & $(590)$ & $1.4 \times 10^{-6}$ & 0.28 & 0.17 \\
\hline 164353 & $67 \mathrm{Oph}$ & B5 Ib & 0.081 & -6.8 & 37.3 & 1.5 & $2.4210^{-10}$ & 500 & $1.210^{-7}$ & 0.07 & 0.03 \\
\hline 15497 & & B6Ia & 0.11 & -8.2 & 77.8 & 1.5 & $1.3110^{-9}$ & $(600)$ & $7.910^{-7}$ & 0.15 & 0.08 \\
\hline 183143 & & B7Ia & $<0.17$ & -8.3 & 88.2 & 1.5 & $<8.49 \times 10^{-10}$ & $(600)$ & $<5.1 \times 10^{-7}$ & $<0.09$ & $<0.05$ \\
\hline 34085 & $\beta$ Ori & B81a & $\left\{\begin{array}{l}2.20 \\
5.05\end{array}\right.$ & -8.9 & 135 & 1.45 & $\begin{array}{l}1.63 \times 10^{-9} \\
2.65 \times 10^{-9}\end{array}$ & 530 & $\left.\begin{array}{l}8.6 \times 10^{-7} \\
1.4 \times 10^{-6}\end{array}\right\}$ & 0.10 & 0.08 \\
\hline 166937 & $\mu \mathrm{Sgr}$ & B8Ia & 0.29 & -8.7 & 123 & 1.45 & $2.01 \times 10^{-9}$ & (530) & $1.1 \times 10^{-6}$ & 0.12 & 0.08 \\
\hline 208501 & 13 Cep & B8Ib & $<0.20$ & -6.6 & 46.9 & 1.45 & $<4.71 \times 10^{-10}$ & $(530)$ & $<2.5 \times 10^{-7}$ & $<0.19$ & $<0.05$ \\
\hline 21291 & & B9 Ia & 0.26 & -7.6 & 84.2 & 1.44 & $8.28 \times 10^{-10}$ & $(500)$ & $4.1 \times 10^{-7}$ & 0.12 & 0.05 \\
\hline 202850 & $\sigma$ Cyg & B9 Iab & $<0.13$ & -6.8 & 58.3 & 1.44 & $<5.25 \times 10^{-10}$ & $(500)$ & $<2.6 \times 10^{-7}$ & $<0.15$ & $<0.04$ \\
\hline 212953 & $4 \mathrm{Lac}$ & B9 Iab & $<0.11$ & -6.5 & 50.8 & 1.44 & $<4.92 \times 10^{-10}$ & $(500)$ & $<2.5 \times 10^{-7}$ & $<0.19$ & $<0.05$ \\
\hline 21389 & & AOIa & 0.73 & -7.1 & 84.7 & 1.44 & $1.40 \times 10^{-9}$ & $(300)$ & $4.2 \times 10^{-7}$ & 0.11 & 0.08 \\
\hline 87737 & $n$ Leo & $\mathrm{AOIb}$ & 0.054 & -5.5 & 40.5 & 1.44 & $1.55 \times 10^{-10}$ & $(300)$ & $4.7 \times 10^{-8}$ & 0.06 & 0.02 \\
\hline 12953 & & $\mathrm{AlIa}$ & 0.23 & -8.0 & 136 & 1.4 & $2.68 \times 10^{-9}$ & $(390)$ & $8.0 \times 10^{-7}$ & 0.10 & 0.10 \\
\hline 14489 & 9 Per & $\mathrm{A} 2 \mathrm{Ia}$ & 0.17 & -7.5 & 115 & 1.4 & $1.73 \times 10^{-9}$ & $(300)$ & $5.2 \times 10^{-7}$ & 0.10 & 0.07 \\
\hline 197345 & $\alpha$ Cyg & $\mathrm{A} 2 \mathrm{Ia}$ & 2.59 & -7.9 & 138 & 1.4 & $2.45 \times 10^{-9}$ & 280 & $6.9 \times 10^{-7}$ & 0.09 & 0.09 \\
\hline $1737 \varepsilon$ & & A5Ia & 0.05 & -7.8 & 156 & 1.35 & $1.15 \times 10^{-9}$ & (280) & $3.2 \times 10^{-7}$ & 0.05 & 0.04 \\
\hline 193237 & P Cyg & B1 I & 6.51 & -10.4 & 89.2 & 1.7 & $5.0 \times 10^{-8}$ & 300 & $1.5 \times 10^{-5}$ & 0.20 & 2.7 \\
\hline 152236 & $\zeta^{1} \mathrm{Sco}$ & B1 I & $8.26^{\dagger}$ & -10.2 & 81.4 & 1.30 & $2.91 \times 10^{-8}$ & 500 & $1.46 \times 10^{-5}$ & 0.38 & 1.74 \\
\hline 152236 & $\zeta^{1} \mathrm{Sco}$ & B1 I & $9.79^{\ddagger}$ & -10.2 & 81.4 & 1.35 & $2.79 \times 10^{-8}$ & 500 & $1.40 \times 10^{-5}$ & 0.36 & 1.67 \\
\hline
\end{tabular}

* $11.3 \mu$ flux

$\dagger^{1.65 \mu \text { flux }}$

$\neq_{2.2 \mu \text { flux }}$ 
supergiants likely to be detectable at $10 \mathrm{GHz}$ with present systems is $\alpha \mathrm{Cyg}$, for which a $10 \mathrm{GHz}$ flux of $2.2 \mathrm{mJy}$ is predicted.

\section{INFRARED OBSERVATIONS OF Of AND OE STARS}

As a preliminary survey, we have observed 10 Of and Oe stars of various spectral types. In all but two cases, only upper limits were obtained at $10 \mu \mathrm{m}$. The greater difficulty associated with detecting $\mathrm{O}$ stars at $10 \mu \mathrm{m}$ is due to their intrinsically fainter brightness at this wavelength compared with the B supergiants. A more extensive survey of $\mathrm{O}$ stars, extending to a significantly fainter $10 \mu \mathrm{m}$ flux limit, is planned. Table 5 presents our photometry. Column (1) gives the HD number of the star observed and column (2) gives the spectral type, taken primarily from Conti and Leep (1974), with the spectral types of HD 39680 and HD 60848 taken from Walborn (1973) and Morgan, Code, and Whitford (1955), respectively. Columns (3) and (4) give the $V$-magnitude and $(B-V)$ color, selected from the catalog of Blanco et al. (1968), except for HD 60848, which is from Guetter (1974). HD 60848 = BN Gem is an irregular variable, and the visual magnitudes given by Morgan, Code, and Whitford (1955), D. A. Allen (1973), and Guetter (1974) span a range of 0.8 mag. Columns (5) through (7) of Table 5 give our photometry at $2.2,3.6$, and $10 \mu \mathrm{m}$ in the same form as described for Table 1 .

In contrast to the case of the B and A supergiants, excess emission at $3.6 \mu \mathrm{m}$ is found for several Of stars. This is due to the fact that a given free-free flux at a particular wavelength (due to a given mass loss rate) will be more noticeable against the stellar continuum of an intrinsically fainter star. The $3.6 \mu \mathrm{m}$ magnitude for the case of no excess can be predicted using equation (2), and it is found that, of the eight stars observed at $3.6 \mu \mathrm{m}$, only HD 17603, $\lambda$ Ori, and HD 193514 exhibit no excess [these three stars are all of type $\mathrm{O}(\mathrm{f})$ or $\mathrm{O}((\mathrm{f}))$, indicating weaker extended emission regions than for the Of and Oe stars; cf. Walborn 1973 and Conti and Leep 1974]. Of the two stars not observed at $3.6 \mu \mathrm{m}$, the $2.2 \mu \mathrm{m}$ magnitude measured by D. A. Allen (1973) for HD 108 indicates an excess at that wavelength, while our $2.2 \mu \mathrm{m}$ photometry of $\lambda$ Cep indicates no excess. Our $10 \mu \mathrm{m}$ upper limit for $\lambda$ Cep lies just fainter than the blackbody tail flux level, so that no mass loss is detectable from this star, a surprising result in view of the fact that it is a high-luminosity Oef star. We therefore plan to reobserve this object.

The three Oe stars observed all show excess emission at $3.6 \mu \mathrm{m}$, and two of the three show excess emission at $2.2 \mu \mathrm{m}$. D. A. Allen (1973) obtained $K=6.65$ for HD 60848, whereas our photometry in 1974 February gave $K=6.85$. This difference is probably real, in view of the fact that the star is a visual variable. However, photometry obtained in 1976 May at Mount Lemmon using an InSb photovoltaic detector gave [1.65] $=7.05 \pm 0.01,[2.3]=$ $6.87 \pm 0.01$, and $[3.5]=6.67 \pm 0.01$; in excellent agreement with our 1974 February photometry which gave $[2.2]=6.85 \pm 0.05$ and $[3.6]=6.58 \pm 0.20$, indicating no significant change in the infrared during that time interval.

We have applied to our infrared observations of the $O$ stars the velocity law and theory described in $\S$ IV, in order to obtain mass loss rates. However, it would be desirable to obtain an independent derivation of the velocity law for an early-type $\mathrm{O}$ star by means of a radio detection. The best candidate for this is $\zeta$ Pup (O4f), which Johnson (1968) has observed at $2.2 \mu \mathrm{m}$. His value of [2.2] $=2.93$, in conjunction with $V=2.26$ and $(B-V)=-0.28$, implies an excess of $0.16 \mathrm{mag}$ at $2.2 \mu \mathrm{m}$, corresponding to $5.63 \mathrm{Jy}$ of excess flux. A mass loss rate of $\dot{M}=3.5 \times$ $10^{-6} M_{\odot} \mathrm{yr}^{-1}$ is derived, in fair agreement with the derivation from Copernicus ultraviolet spectra of the same star by Lamers and Morton (1976) of $\dot{M}=(7 \pm 3) \times 10^{-6} M_{\odot} \mathrm{yr}^{-1}$. This may indicate that the P Cygni velocity law is applicable to $\mathrm{O}$ stars also. Certainly the mass loss rates determined for the same star from the excess fluxes at different wavelengths are consistent with each other for all of the $O$ stars except HD 108 (see Table 6). The mass loss rate determined for $\zeta$ Pup from the $2.2 \mu \mathrm{m}$ data predicts a $10 \mathrm{GHz}$ radio flux of $2.5 \mathrm{mJy}$. Radio observations of this star would thus appear feasible. An upper limit of $15 \mathrm{mJy}$ at $8.87 \mathrm{GHz}$ has been set for $\zeta$ Pup by A. E. Wright (1975, private communication).

TABLE 5

PHOTOMETRY OF Oe AND Of STARS

\begin{tabular}{|c|c|c|c|c|c|c|}
\hline HD & Spectral Type & $V$ & $(B-V)$ & {$[2.2]$} & [3.6] & [10] \\
\hline 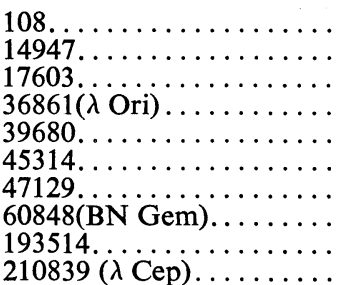 & $\begin{array}{l}\text { O7 If } \\
\text { O5.5f } \\
\text { O8.5 I(f) } \\
\text { O8 III((f)) } \\
\text { O9 Vpe var } \dagger \\
\text { OBe } \\
\text { O7.5 IIIf } \\
\text { O8 V:pe } \\
\text { O7.5 III(f) } \\
\text { O6ef }\end{array}$ & $\begin{array}{l}7.40 \\
7.98 \\
8.45 \\
3.39 \\
7.99 \\
6.64 \\
6.05 \\
6.85 \\
7.42 \\
5.04\end{array}$ & $\begin{array}{l}+0.18 \\
+0.46 \\
+0.64 \\
-0.19 \\
+0.02 \\
+0.15 \\
+0.06 \\
-0.21 \\
+0.45 \\
+0.25\end{array}$ & $\begin{array}{l}6.71^{*} \\
7.02(10) \\
6.80(09) \\
3.95(01) \\
6.72(09) \\
6.38(05) \\
5.98(06) \\
6.85(05) \\
6.22(06) \\
4.49(01)\end{array}$ & $\begin{array}{l}6.39(15) \\
6.56(16) \\
4.07(03) \\
6.23(12) \\
5.93(07) \\
5.62(10) \\
6.58(20) \\
6.30(16) \\
\ldots\end{array}$ & $\begin{aligned}> & 6.15 \\
> & 4.82 \\
> & 4.49 \\
& 3.91(21) \\
> & 4.45 \\
> & 5.00 \\
& 4.70(33) \\
> & 3.54 \\
> & 4.6 \\
> & 4.44\end{aligned}$ \\
\hline
\end{tabular}

* D. A. Allen (1973).

$\uparrow$ Spectral type estimated following Frost and Conti (1975). 
Lamers and Morton (1976) have derived a wind temperature of $2 \times 10^{5} \mathrm{~K}$ for $\zeta$ Pup and in addition have found that at $R \approx 1.7 R_{c}$ the velocity $v(R)$ is equal to $0.25 v_{\infty}$, with the velocity law beyond this point similar to the standard radiative acceleration law (14). Inside $R \approx 1.7 R_{c}$, they could not define the velocity law, however. We have therefore experimented with various types of velocity laws inside $R \approx 1.7 R_{c}$ (which were constrained to obey the same boundary conditions as in $\S \mathrm{V}$ ) in order to find one that, with a wind temperature of $2 \times 10^{5} \mathrm{~K}$ and a mass loss rate of $5.5 \times 10^{-6} M_{\odot} \mathrm{yr}^{-1}$, would give rise to a flux of 5.6 Jy at $2.2 \mu \mathrm{m}$. The velocity law which achieved this was

$$
\begin{aligned}
& v(R)=v_{\infty}\left[1-\left(1-X_{c}{ }^{0.84}\right)\left(\frac{R_{c}}{R}\right)^{0.19}\right]^{0.595} ; \frac{R}{R_{c}}<1.7, \\
& v(R)=v_{\infty}\left[0.25+0.75\left[1-\left(\frac{1.7 R_{c}}{R}\right)\right]^{1 / 2}\right] ; \frac{R}{R_{c}} \geq 1.7 .
\end{aligned}
$$

Since only one infrared flux data point exists for $\zeta$ Pup, the validity of either the P Cygni law (20) or the "hot" $\zeta$ Pup law (21) cannot be distinguished at present. However, future observations of $\zeta$ Pup in the $10-20 \mu \mathrm{m}$ region should be able to distinguish between the two laws, since the "hot" $\zeta$ Pup law predicts a significantly flatter spectrum in this region. Quantitatively, the P Cygni velocity law (20) predicts $2.3 \mathrm{Jy}$ at $10 \mu \mathrm{m}$ from $\zeta$ Pup, whereas the hot $\zeta$ Pup law (21) predicts $5.1 \mathrm{Jy}$ at $10 \mu \mathrm{m}$. In the remainder of this section, we will use the P Cygni velocity law (20) for the mass loss rate determinations for our small sample of $O$ stars.

Columns (1)-(9) of Table 6 list the usual stellar parameters defined earlier. In deriving the $O$ star parameters, we have used the effective temperature scale of Conti (1973) and Panagia (1973). Columns (10) and (11) list the wavelength and the excess flux at that wavelength used in the particular determination of $\dot{M} / v_{\infty}$, which is given in column (12). Column (13) lists the value of the terminal velocity $v_{\infty}$ for each star, taken directly or interpolated from the survey of Snow and Morton (1976). We have assumed that helium atoms are twice ionized in stellar winds from stars with effective temperatures $T \geq 40,000 \mathrm{~K}$, so that $\mu=1.26, Z^{2}=1.27$, and $\gamma=1.09$. For all stars cooler than $40,000 \mathrm{~K}$ we have assumed that helium is singly ionized, giving $\mu=1.26, Z^{2}=1.0$, and $\gamma=1.0$.

From our photometry of $\lambda$ Ori we find a small excess of $0.134 \mathrm{Jy}$ at $10 \mu \mathrm{m}$, in good agreement with the excess flux of $0.147 \mathrm{Jy}$ at $10 \mu \mathrm{m}$ that can be derived from the photometry of Gehrz, Hackwell, and Jones (1974). In our mass loss determination for $\lambda$ Ori, we have adopted a mean $10 \mu \mathrm{m}$ excess of $0.14 \mathrm{Jy}$.

No mass loss rate determination is quoted for HD 47129 (Plaskett's star). This star has significant excess flux at both 3.6 and $10 \mu \mathrm{m}$. However, the star is a massive spectroscopic binary with components having a mass ratio of approximately unity (Hutchings and Cowley 1976). Hutchings and Cowley have found evidence for both mass loss from the system and mass transfer within the system, the apparent relative strength of these phenomena varying with orbital phase. Since further infrared, optical and ultraviolet observations are planned, a discussion of the system will be deferred until then.

The 2.2 and 3.6 $\mu \mathrm{m}$ excess fluxes from HD 39680 and the 1.65, 2.3, and $3.5 \mu \mathrm{m}$ excess fluxes from HD 60848 give values of $\dot{M} / v_{\infty}$ in good agreement with each other. However, the values of $\dot{M} /\left(L / v_{\infty} c\right)$ derived for these two Oe stars (cf. § VII) are significantly larger than unity. One possible reason for this may be that the value of $v_{\infty}=1200$ $\mathrm{km} \mathrm{s}^{-1}$ adopted for both stars may be too large. Another possibility may be that the $U B V$ data used to derive intrinsic infrared magnitudes may have been too faint, since one star is a known variable and the other is at least a spectrum variable. Finally, it may be that a more complicated model than spherically symmetric mass loss is required for the Oe stars, since the work of Conti and Leep (1974) indicates the presence of a rapidly rotating gaseous envelope around these stars.

The mass loss rates found for $\zeta$ Pup and HD 14947 cannot be directly compared with those found by Conti and Frost (1977) (cf. $\S \mathrm{V}$ ), since different velocity laws are assumed. At the time of writing it is not known by how much the P Cygni velocity law (20) would decrease the mass loss rates derived from $\mathrm{H} \alpha$ fluxes by Conti and Frost.

\section{THE NATURE OF THE MASS LOSS MECHANISM FOR EARLY-TYPE STARS}

Lucy and Solomon (1970) proposed that the mass loss observed from O and B stars was due to radiation pressure acting through ultraviolet resonance-line absorption. Castor, Abbott, and Klein (1975) have extended and modified this idea by including the effects of lines other than the resonance-line transitions of the dominant ions. A full review of radiation pressure theories and competing coronal mass loss theories is given by Castor, Abbott, and Klein (1976).

Lucy and Solomon (1970) predicted that the mass loss rate from OB stars would be that due to only a few principal resonance lines, each line contributing an upper limit of $L / c^{2}$ to the mass loss rate. The model of Castor, Abbott, and Klein (1975), taking account of many more transitions, predicts that the mass loss rate may approach the upper limit for continuum radiation pressure, $L / v_{\infty} c$. Therefore the model of Castor, Abbott, and Klein predicts mass loss rates of up to approximately 100 times larger than predicted by Lucy and Solomon. In the 


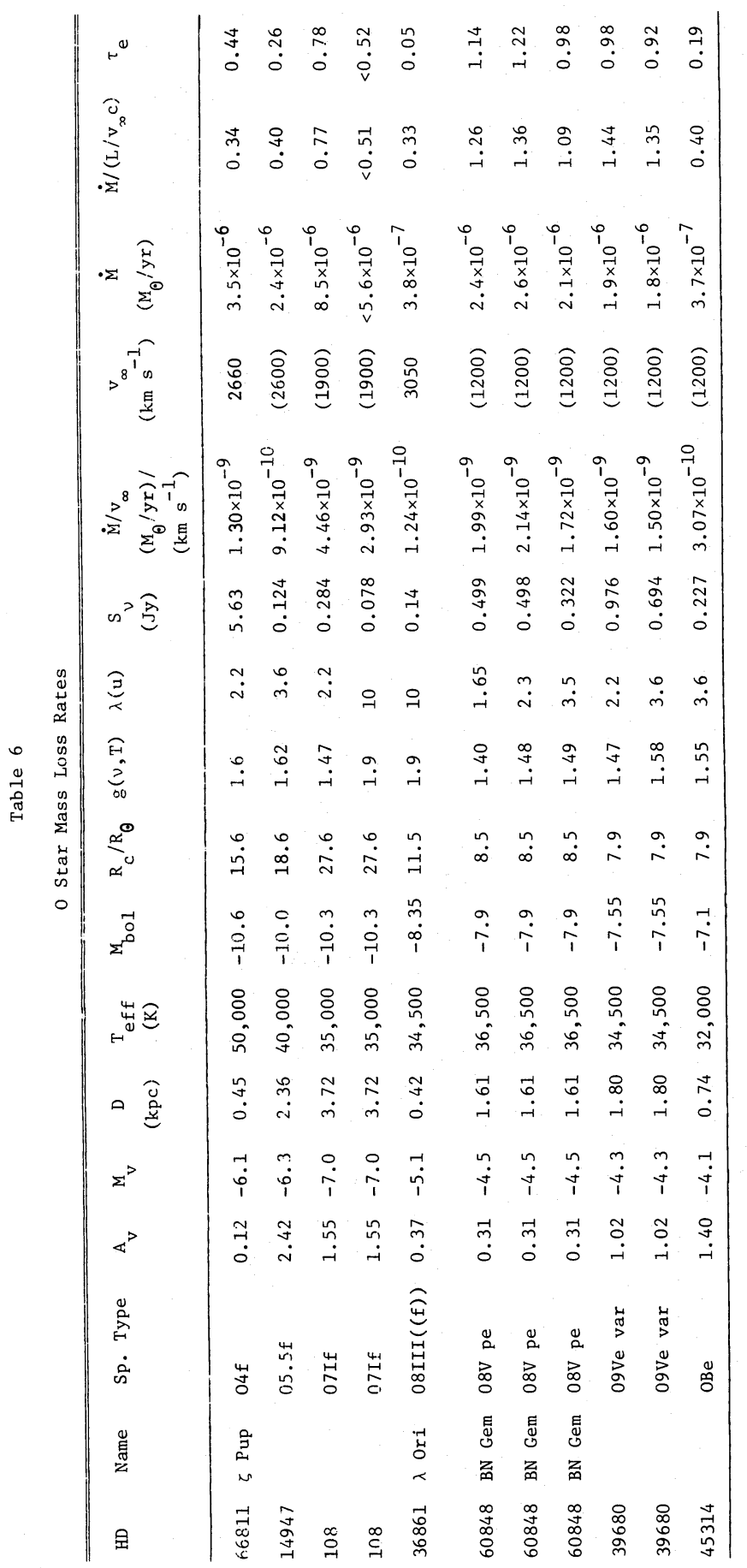




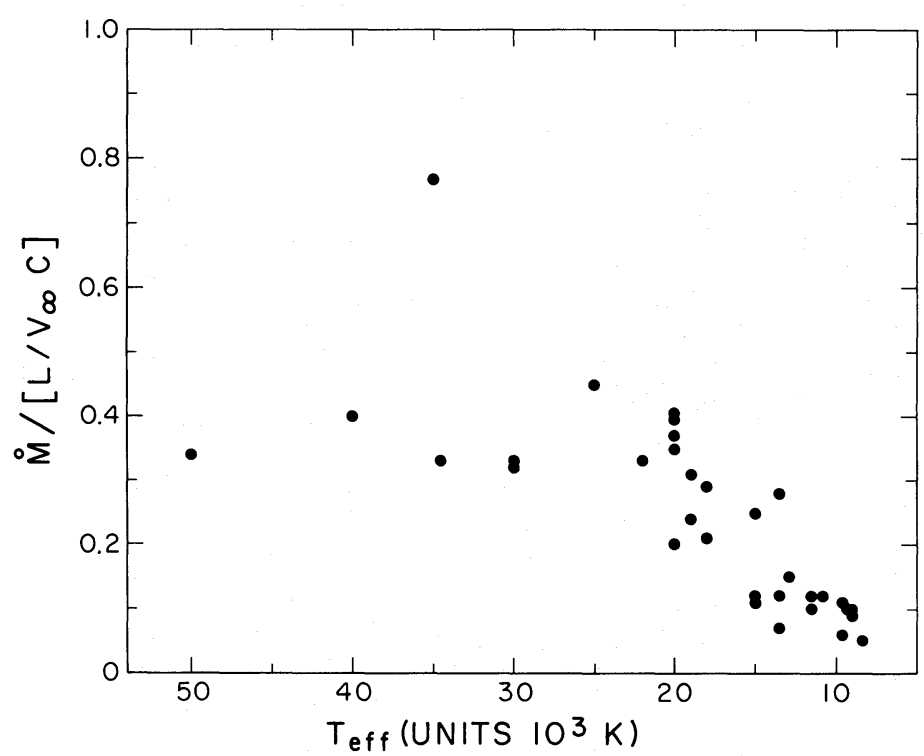

FIG. 6. - The ratio of the mass loss rates $\dot{M}$ derived in Tables 4 and 6 to the upper limits for mass loss by radiation pressure, $\left(L / v_{\infty} c\right)$, plotted versus effective temperature $T_{\text {eff }}$ for $33 \mathrm{OBA}$ supergiants and Of stars.

penultimate columns of Tables 4 and 6, we have listed for each star $\dot{M} /\left(L / v_{\infty} c\right)$, the ratio of the observed mass loss rate to the upper limit for radiation pressure. In Figure 6 this ratio is plotted as a function of stellar effective temperature. For $\delta, \zeta, \epsilon$, and $\kappa$ Ori we have plotted the points corresponding to the lower of the values of $v_{\infty}$ discussed in $\S \mathrm{V}$, and for $\beta$ Ori we have taken the mean of the two determinations of $\dot{M}$. We have omitted the Oe stars from Figure 6, since rotation probably influences their mass loss rates (§ VI). Two conclusions are apparent from Figure 6. (1) The values taken by the ratio are consistent with the theory of Castor, Abbott, and Klein (1975) and are too large to be explained by the original resonance-line theory of Lucy and Solomon (1970). (2) For stellar effective temperatures in the range $2 \times 10^{4}-5 \times 10^{4} \mathrm{~K}$, the ratio is approximately constant at a value of $\sim 0.4$, apart from the extreme Of star HD 108. On the other hand, for effective temperatures less than $2 \times 10^{4} \mathrm{~K}$, the value of the ratio declines with decreasing temperature. This is presumably due to the fact that, as stellar temperature decreases, the spectral region containing lines suitable for the radiation-pressure mechanism intercepts less and less stellar flux. Figure 6 indicates that, for the stars investigated here, radiation pressure is probably responsible for mass loss in the entire spectral type range O4-A5.

Recently Hutchings (1976a) has estimated mass loss rates for OB supergiants by means of a qualitative relationship between the number of spectroscopic mass loss indicators and the mass loss rate, the relationship being calibrated by stars with known mass loss rates. The present paper has 18 stars in common with that of Hutchings $(1976 a)$. For six stars, the mass loss rates found here and by Hutchings agree within a factor of 3 , but for 12 stars the mass loss rates differ by factors of between 3 and 20. One of the reasons for this disagreement is probably that several of the mass loss rates used for calibration by Hutchings are much too large. For instance, a mass loss rate of $3.5 \times 10^{-4} M_{\odot} \mathrm{yr}^{-1}$ is quoted for $\mathrm{P}$ Cyg, which is 23 times larger than the value found here and exceeds the upper limit for radiation pressure by a factor of 5. Indeed, many of the mass loss rates derived by Hutchings (1976a) exceed the upper limit for mass loss by radiation pressure.

In Figure 7 we have plotted for each star the value of $M_{\text {bol }}$ versus $\log \left(10^{8} \dot{M}\right)$, where $\dot{M}$ is in units of $M_{\odot} \mathrm{yr}^{-1}$. We have given different symbols to the $\mathrm{O}$ stars and B stars, since a natural gap of $5000 \mathrm{~K}$ exists between 09.5 and B0 supergiants. We have also given different symbols to B0-B6 and B7-A 5 stars, since Figure 3 and the work of Rosendhal (1973) indicated that there might be a separation between these two groups. However, Figure 7 reveals that the B0-A5 stars define a single relation, while the $\mathrm{O}$ stars define another but parallel relation, in the sense that the $\mathrm{O}$ stars have less mass loss per unit luminosity. Least-squares fits to the two groups of stars give the following relations:

For the O stars (excluding HD 108),

$$
\dot{M}=6.8 \times 10^{-13} L^{1.10 \pm 0.06} M_{\odot} \mathrm{yr}^{-1} .
$$

For the B and A supergiants,

where $L$ is the units of $L_{\odot}$.

$$
\dot{M}=5 \times 10^{-13} L^{1.20 \pm 0.08} M_{\odot} \mathrm{yr}^{-1}
$$




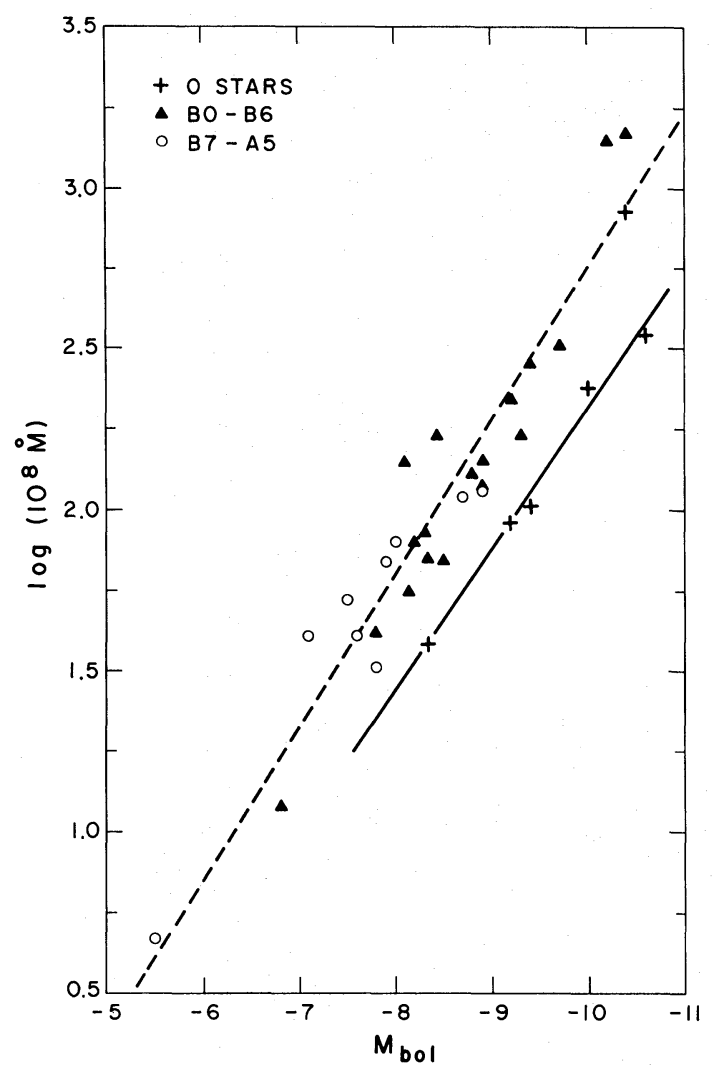

FIG. 7.-The quantity $\log _{10}\left(10^{8} \dot{M}\right)$ versus absolute bolometric magnitude $M_{\text {bol }}$ for 33 OBA supergiants and Of stars. The mass loss rates $\dot{M}$ are in units of $M_{\odot} \mathrm{yr}^{-1}$ and are those derived in Tables 4 and 6 . The B0-A5 supergiants define one relation and the Of stars and $O$ supergiants define another but parallel relation, in the sense that the $O$ stars have less mass loss per unit stellar luminosity. The straight lines show the least-squares fits to the data for the two groups of stars.

The power dependence of $\dot{M}$ upon $L$ in relation (22) for the $\mathrm{O}$ stars is consistent with the range predicted by Castor, Abbott, and Klein (1976). No predictions have as yet been made for B and A supergiants, but Figure 7 indicates that a similar relation holds. Comparison of Figure 7 with Figure 6 shows that, although the fraction of radiation momentum converted into mass loss momentum declines with declining effective temperature for the B and A supergiants, mass loss per unit stellar luminosity remains constant, due to the corresponding decline with decreasing effective temperature of the terminal wind velocity of the later spectral type stars.

The electron-scattering optical depth, $\tau_{e}$, from the photosphere $R_{c}$ to infinity, contributed by the stellar wind of a star undergoing mass loss is given by

$$
\tau_{e}=\int_{R_{c}}^{\infty} \sigma_{e} n_{e}(R) d R,
$$

where $\sigma_{e}$ is the Thomson scattering cross section and $n_{e}(R)$ is the electron density. For the velocity law (20), $n_{e}(R)$ is given by

$$
n_{e}(R)=\frac{\gamma A}{R^{2}\left[1-\left(1-1.91 \times 10^{8} X_{c}^{2.38}\right) R_{c} / R\right]^{0.21} \operatorname{dex}\left[-1.74 R_{c} / R\right]}
$$

where $A=\dot{M} / 4 \pi \mu m_{\mathrm{H}} v_{\infty}$.

Substituting equation (25) into (24), we find that $\tau_{e}$ is independent of $X_{c}$ for the range of $X_{c}$ appropriate for the stars under investigation here $\left(X_{c} \approx 10^{-6}-10^{-10}\right)$. Thus, on integrating we obtain

$$
\tau_{e}=21.35 \sigma_{e} \gamma A / R_{c},
$$

where $\sigma_{e}, A$, and $R_{c}$ are in cgs units. 
The value of $\tau_{e}$ derived for each star is given in the last columns of Tables 4 and 6 . All of the supergiants have $\tau_{e}<0.2$, except for $\zeta^{1}$ Sco and P Cyg, for which $\tau_{e}=1.7$ and 2.7, respectively. These values may be compatible with the spectra of these two stars, since practically all of the lines in the spectrum of P Cyg, and many of the lines in the spectrum of $\zeta^{1}$ Sco, show emission (Hutchings 1968, 1969), indicating that they originate above the photosphere, in the expanding atmosphere. A lower value of $\tau_{e}$ would thus be appropriate for these lines. For instance, a line from P Cyg originating from 1.3 $R_{c}$ would experience an electron-scattering optical depth $\tau_{e}$ to infinity of 0.81 and at $1.5 R_{c}, \tau_{e}$ is only 0.49 .

The theory of Castor, Abbott, and Klein (1975) predicts that the energy distribution of an early-type star undergoing mass loss should be closely similar to that predicted by a static plane-parallel model atmosphere, with the principal difference being that the existence of a halo due to the electron-scattering envelope increases the apparent effective radius of the star. The value of $\tau_{e}=0.44$ obtained here for $\zeta$ Pup using the P Cygni velocity law (20) is consistent with the value of $\tau_{e} \approx 1 / 3$ required by Holm and Cassinelli (1976) to obtain agreement between the observed energy distribution and plane-parallel models with an effective temperature of $\sim 42,000 \mathrm{~K}$. The "hot" velocity law (21) predicts that $\tau_{e}=0.17$ for $\zeta$ Pup.

In summary, the mass loss rates obtained in this paper for OBA supergiants and Of stars are consistent with radiation pressure being the driving mechanism of the mass loss flows.

We would like to thank Dr. T. P. Snow for communication in advance of publication of the results of the Copernicus mass loss survey. We would also like to thank Dr. K. Nandy for communication of unpublished TD-1 data on supergiant ultraviolet energy distributions. We thank Dr. P. R. Schwartz and Dr. J. H. Spencer for communication in advance of publication of their $3.3 \mathrm{~mm}$ data for P Cyg. We are grateful to C. V. Kunasz and P. B. Kunasz for the loan of their spherical transfer programs and for helpful discussions on their use. We acknowledge useful discussions with D. C. Abbott, J. I. Castor, P. S. Conti, S. A. Frost, and D. Van Blerkom. It is a pleasure to thank Drs. W. A. Stein and N. J. Woolf for observing time at Mount Lemmon. Infrared astronomy at Berkeley during the period of the observations was supported by the National Science Foundation under grant GP-31592X. Research at J.I.L.A. was supported by NSF grant AST 72-05026 A03.

\section{APPENDIX}

\section{THEORETICAL INTRINSIC COLORS FOR BLACKBODY ENERGY DISTRIBUTIONS}

Suppose we wish to calculate the intrinsic color index of a star between wavelengths $\lambda_{1}$ and $\lambda_{2} \mu \mathrm{m}$. By definition, the magnitude at wavelength $\lambda_{i} \mu \mathrm{m}$ is

$$
\left[\lambda_{i}\right]=-2.5 \log _{10}\left[F\left(\lambda_{i}\right) / F_{0}\left(\lambda_{i}\right)\right],
$$

where $F\left(\lambda_{i}\right)$ is the stellar flux at $\lambda_{i}$, and $F_{0}\left(\lambda_{i}\right)$ is the flux equivalent to zero magnitude, in $\mathrm{W} \mathrm{cm}^{-2} \mu \mathrm{m}^{-1}$.

Assuming that the star emits as a blackbody of temperature $T$,

$$
\left[\lambda_{i}\right]=-2.5 \log _{10}\left[B\left(\lambda_{i}, T\right) / F_{0}\left(\lambda_{i}\right)\right],
$$

where $B\left(\lambda_{i}, T\right)$ is the Planck function at temperature $T$. Thus

$$
\left[\lambda_{1}\right]-\left[\lambda_{2}\right]=-2.5 \log _{10}\left[\frac{B\left(\lambda_{1}, T\right) F_{0}\left(\lambda_{2}\right)}{B\left(\lambda_{2}, T\right) F_{0}\left(\lambda_{1}\right)}\right]+k\left(\lambda_{1}, \lambda_{2}\right),
$$

where $k\left(\lambda_{1}, \lambda_{2}\right)$ is a constant included to satisfy the convention that $\mathrm{A} 0$ stars have color indices equal to zero. We have derived the intrinsic color relations below, in which $T_{4}$ denotes stellar effective temperature in units of $10^{4} \mathrm{~K}$. Values of $F_{0}(\lambda)$ for infrared wavelengths are taken from Cohen (1973) and at $B$ and $V$ are $6.61(-12)$ and 3.80 $(-12) \mathrm{W} \mathrm{cm}^{-2} \mu \mathrm{m}^{-1}$, respectively (C. W. Allen 1973)

$$
\begin{aligned}
(B-V)_{0} & =2.5 \log _{10}\left[\frac{\exp \left(3.27 / T_{4}\right)-1}{\exp \left(2.62 / T_{4}\right)-1}\right]-0.77, \\
(V-[2.2])_{0} & =2.5 \log _{10}\left[\frac{\exp \left(2.62 / T_{4}\right)-1}{\exp \left(0.654 / T_{4}\right)-1}\right]-2.92, \\
(V-[3.6])_{0} & =2.5 \log _{10}\left[\frac{\exp \left(2.62 / T_{4}\right)-1}{\exp \left(0.394 / T_{4}\right)-1}\right]-3.63,
\end{aligned}
$$




$$
\begin{aligned}
([3.6]-[10])_{0} & =2.5 \log _{10}\left[\frac{\exp \left(0.394 / T_{4}\right)-1}{\exp \left(0.144 / T_{4}\right)-1}\right]-1.24, \\
([3.6]-[11.3])_{0} & =2.5 \log _{10}\left[\frac{\exp \left(0.394 / T_{4}\right)-1}{\exp \left(0.127 / T_{4}\right)-1}\right]-1.39, \\
([3.6]-[18])_{0} & =2.5 \log _{10}\left[\frac{\exp \left(0.394 / T_{4}\right)-1}{\exp \left(0.080 / T_{4}\right)-1}\right]-1.92 .
\end{aligned}
$$

The various constants are computed to give an index of zero for $T_{4}=0.96$.

\section{REFERENCES}

Allen, C. W. 1973, Astrophysical Quantities (3d ed.; London: Athlone Press).

Allen, D. A. 1973, M.N.R.A.S., 161, 145.

Altenhoff, W. J., Braes, L. L. E., Olnon, F. M., and Wendker, H. J. 1976, Astr. Ap., 46, 11.

Blanco, V. M., Demers, S., Douglas, G. G., and FitzGerald, M. P. 1968, Pub. US Naval Obs., 21.

Brunet, J. P. 1975, Astr. Ap., 43, 345.

Carrasco, L., Strom, S. E., and Strom, K. M. 1973, Ap. J., 182 95.

Cassinelli, J. P., and Castor, J. I. 1973, Ap. J., 179, 189.

Castor, J. I., Abbott, D. C., and Klein, R. I. 1975, Ap. J., 195, 157.

1976, in Colloques Internationaux du C.N.R.S., No. 250, Physique des Mouvements dans les Atmosphères Stellaires, ed. R. Cayrel and M. Steinberg (Paris, C.N.R.S.), p. 363.

Code, A. D., Davis, J., Bless, R. C., and Hanbury Brown, R. 1976, Ap. J., 203, 417.

Cohen, M. 1973, M.N.R.A.S., 161, 85.

Cohen, M., Barlow, M. J., and Kuhi, L. V. 1975, Astr. Ap., 40, 291.

Conti, P. S. 1973, Ap. J., 179, 181.

Conti, P. S., and Alschuler, W. R. 1971, Ap. J., 170, 325.

Conti, P. S., and Frost, S. A. 1977, Ap. J., 212, 728.

Conti, P. S., and Leep, E. M. 1974, Ap. J., 193, 113.

Fernie, J. D. 1969, Pub. A.S.P., 81, 168.

Frost, S. A., and Conti, P. S. 1975, in Symposium No. 70 , Be and Shell Stars, ed. A. Slettebak (Dordrecht: Reidel), in press.

Gehrz, R. D., Hackwell, J. A., and Jones, T. W. 1974, Ap. J., 191, 675.

Guetter, H. H. 1974, Pub. A.S.P., 86, 795.

Hackwell, J. A., and Gehrz, R. D. 1974, Ap. J., 194, 49.

Hackwell, J. A., Gehrz, R. D., and Smith, J. R. 1974, Ap. J., $192,383$.

Harris, D. H. 1973, in IAU Symposium No. 52, Interstellar Dust and Related Topics, ed. J. M. Greenberg and H. C. van de Hulst (Dordrecht: Reidel), p. 31.

Hearn, A. G. 1975, Astr. Ap., 40, 277.

Holm, A. V., and Cassinelli, J. P. 1976, preprint.

Humphries, C. M., Nandy, K., and Kontizas, E. 1975, Ap. J., 195, 111.
Hutchings, J. B. 1968, M.N.R.A.S., 141, 219.

. 1969, M.N.R.A.S., 144, 235.

-. 1970, M.N.R.A.S., 147, 161.

- $1976 a, A p . J ., 203,438$. 1976b, Ap. J.' (Letters), 204, L99.

Hutchings, J. B., and Cowley, A. P. 1976, Ap. J., 206, 490.

Johnson, H. L. 1968, in Nebulae and Interstellar Matter, ed. B. M. Middlehurst and L. H. Aller (Chicago: University of Chicago Press), p. 191

Karzas, T. A., and Latter, R. 1961, Ap. J. Suppl., 6, 167.

Kuan, P., and Kuhi, L. V. 1975, Ap. J., 199, 148.

Kunasz, P. B., and Hummer, D. G. 1974, M.N.R.A.S., 166, 19.

Kunasz, P. B., and Kunasz, C. V. 1977, in preparation.

Lamers, H. J. G. L. M., and Morton, D. C. 1976, Ap. J. Suppl., 32, 715 .

Lee, T. A. 1968, Ap. J., 152, 913.

Lesh, J. R. 1968, Ap. J. Suppl., 17, 371.

Lucy, L. B., and Solomon, P. M. 1970, Ap. J., 159, 879.

Maeder, A., and Rufener, F. 1972, Astr. Ap., 20, 437.

Mihalas, D. 1972, NCAR Technical Note, STR-76.

Morgan, W. W., Code, A. D., and Whitford, A. E. 1955, Ap. J. Suppl., 2, 41.

Morrison, D., and Simon, T. 1973, Ap. J., 186, 193.

Morton, D. C. 1967, Ap. J., 150, 535.

Morton, D. C., and Adams, T. F. 1968, Ap. J., 151, 611.

Panagia, N. 1973, A.J., 78, 929.

Panagia, N., and Felli, M. 1975, Astr. Ap., 39, 1.

Rogerson, J. B., and Lamers, H. J. G. L. M. 1975, Nature, 256, 190.

Rosendhal, J. D. 1973, Ap. J., 186, 909.

Schalen, C. 1975, Astr. Ap., 42, 251.

Schild, R., Neugebauer, G., and Westphal, J. A. 1971, A.J., 76, 237. Schild, R., Peterson, D. M., and Oke, J. B. 1971, Ap. J., 166,

Schultz, G. V., and Wiemer, W. 1975, Astr. Ap., 43, 133.

Schwartz, P. R., and Spencer, J. H. 1977, in preparation.

Snow, T. P., and Morton, D. C. 1976, Ap. J. Suppl., 32, 429.

Walborn, N. R. 1973, A.J., 78, 1967.

Wendker, H. J., Baars, J. W. M., and Altenhoff, W. J. 1973, Nature Phys. Sci., 245, 5.

Wright, A. E., and Barlow, M. J. 1975, M.N.R.A.S., 170, 41.

Note added in proof.-The terminal velocity of $v_{\infty}=1900 \mathrm{~km} \mathrm{~s}^{-1}$ adopted for HD 108 in Table 6 is similar to the terminal velocity of $1850 \mathrm{~km} \mathrm{~s}^{-1}$ found by Snow and Morton (1976) for HD 151804, a star of similar spectral type and absolute magnitude. However, two other stars of similar spectral type and absolute magnitude, namely HD 152408 and $29 \mathrm{CMa}$, have terminal velocities of $1430 \mathrm{~km} \mathrm{~s}^{-1}$ and $1480 \mathrm{~km} \mathrm{~s}^{-1}$, respectively (Snow and Morton 1976). If $v_{\infty}=1450 \mathrm{~km} \mathrm{~s}^{-1}$ is instead adopted for HD 108, the determination of $\dot{M} / v_{\infty}$ from the $2.2 \mu \mathrm{m}$ excess flux gives $\dot{M}=6.5 \times 10^{-6} M_{\odot} \mathrm{yr}^{-1}$, and $\dot{M} /\left(L / v_{\infty} c\right)$ is reduced from 0.77 to 0.45 . The latter value is then comparable to the values plotted for the other $\mathrm{O}$ stars in Figure 6.

Michael J. BARLow: Joint Institute for Laboratory Astrophysics, University of Colorado, Boulder, CO 80309 MARTIN Cohen: Berkeley Astronomy Department, University of California, Berkeley, CA 94720 